ISSN 1855-3966 (printed edn.), ISSN 1855-3974 (electronic edn.)

ARS MATHEMATICA CONTEMPORANEA 16 (2019) 119-139

https://doi.org/10.26493/1855-3974.1490.eea

(Also available at http://amc-journal.eu)

\title{
The Doyen-Wilson theorem for 3-sun systems*
}

\author{
Giovanni Lo Faro, Antoinette Tripodi \\ Dipartimento di Scienze Matematiche e Informatiche, Scienze Fisiche e Scienze della \\ Terra, Università di Messina, Messina, Italia
}

Received 25 September 2017, accepted 18 April 2018, published online 20 September 2018

\begin{abstract}
A solution to the existence problem of $G$-designs with given subdesigns is known when $G$ is a triangle with $p=0,1$, or 2 disjoint pendent edges: for $p=0$, it is due to Doyen and Wilson, the first to pose such a problem for Steiner triple systems; for $p=1$ and $p=2$, the corresponding designs are kite systems and bull designs, respectively. Here, a complete solution to the problem is given in the remaining case where $G$ is a 3 -sun, i.e. a graph on six vertices consisting of a triangle with three pendent edges which form a 1 -factor.
\end{abstract}

Keywords: 3-sun systems, embedding, difference set.

Math. Subj. Class.: 05B05, 05B30

\section{Introduction}

If $G$ is a graph, then let $V(G)$ and $E(G)$ be the vertex-set and edge-set of $G$, respectively. The graph $K_{n}$ denotes the complete graph on $n$ vertices. The graph $K_{m} \backslash K_{n}$ has vertex-set $V\left(K_{m}\right)$ containing a distinguished subset $H$ of size $n$; the edge-set of $K_{m} \backslash K_{n}$ is $E\left(K_{m}\right)$ but with the $\left(\begin{array}{l}n \\ 2\end{array}\right)$ edges between the $n$ distinguished vertices of $H$ removed. This graph is sometimes referred to as a complete graph of order $m$ with a hole of size $n$.

Let $G$ and $\Gamma$ be finite graphs. A $G$-design of $\Gamma$ is a pair $(X, \mathcal{B})$ where $X=V(\Gamma)$ and $\mathcal{B}$ is a collection of isomorphic copies of $G$ (blocks), whose edges partition $E(\Gamma)$. If $\Gamma=K_{n}$, then we refer to such a design as a $G$-design of order $n$.

A $G$-design $\left(X_{1}, \mathcal{B}_{1}\right)$ of order $n$ is said to be embedded in a $G$-design $\left(X_{2}, \mathcal{B}_{2}\right)$ of order $m$ provided $X_{1} \subseteq X_{2}$ and $\mathcal{B}_{1} \subseteq \mathcal{B}_{2}$ (we also say that $\left(X_{1}, \mathcal{B}_{1}\right)$ is a subdesign (or subsystem $)$ of $\left(X_{2}, \mathcal{B}_{2}\right)$ or $\left(X_{2}, \mathcal{B}_{2}\right)$ contains $\left(X_{1}, \mathcal{B}_{1}\right)$ as subdesign). Let $N(G)$ denote the set of integers $n$ such that there exists a $G$-design of order $n$. A natural question to ask is: given $n, m \in N(G)$, with $m>n$, and a $G$-design $(X, \mathcal{B})$ of order $n$, does exists a $G$-design of order $m$ containing $(X, \mathcal{B})$ as subdesign? Doyen and Wilson were the first to

* Supported by I.N.D.A.M. (G.N.S.A.G.A.).

E-mail addresses: lofaro@unime.it (Giovanni Lo Faro), atripodi@unime.it (Antoinette Tripodi)

() (i) This work is licensed under https://creativecommons.org/licenses/by/4.0/ 
pose this problem for $G=K_{3}$ (Steiner triple systems) and in 1973 they showed that given $n, m \in N\left(K_{3}\right)=\{v: v \equiv 1,3(\bmod 6)\}$, then any Steiner triple system of order $n$ can be embedded in a Steiner triple system of order $m$ if and only if $m \geq 2 n+1$ or $m=n$ (see [3]). Over the years, any such problem has come to be called a "Doyen-Wilson problem" and any solution a "Doyen-Wilson type theorem". The work along these lines is extensive $([1,4,5,6,7,8,9,10,13])$ and the interested reader is referred to [2] for a history of this problem.

In particular, taking into consideration the case where $G$ is a triangle with $p=0,1,2$, or 3 mutually disjoint pendent edges, a solution to the Doyen-Wilson problem is known when $p=0$ (Steiner triple systems, [3]), $p=1$ (kite systems, [9, 10]) and $p=2$ (bull designs, [4]). Here, we deal with the remaining case $(p=3)$ where $G$ is a 3 -sun, i.e. a graph on six vertices consisting of a triangle with three pendent edges which form a 1-factor, by giving a complete solution to the Doyen-Wilson problem for $G$-designs where $G$ is a 3-sun (3-sun systems).

\section{Notation and basic lemmas}

The 3 -sun consisting of the triangle $(a, b, c)$ and the three mutually disjoint pendent edges $\{a, d\},\{b, e\},\{c, f\}$ is denoted by $(a, b, c ; d, e, f)$. A 3 -sun system of order $n$ (briefly, $3 \mathrm{SS}(n))$ exists if and only if $n \equiv 0,1,4,9(\bmod 12)$ and if $(X, \mathcal{S})$ is a $3 \operatorname{SS}(n)$, then $|\mathcal{S}|=\frac{n(n-1)}{12}($ see $[14])$.

Let $n, m \equiv 0,1,4,9(\bmod 12)$, with $m=u+n, u \geq 0$. The Doyen-Wilson problem for 3 -sun systems is equivalent to the existence problem of decompositions of $K_{u+n} \backslash K_{n}$ into 3 -suns.

Let $r$ and $s$ be integers with $r<s$, define $[r, s]=\{r, r+1, \ldots, s\}$ and $[s, r]=\emptyset$. Let $Z_{u}=[0, u-1]$ and $H=\left\{\infty_{1}, \infty_{2}, \ldots, \infty_{t}\right\}, H \cap Z_{u}=\emptyset$. If $S=(a, b, c ; d, e, f)$ is a 3-sun whose vertices belong to $Z_{u} \cup H$ and $i \in Z_{u}$, let $S+i=(a+i, b+i, c+i$; + $i, e+i, f+i)$, where the sums are modulo $u$ and $\infty+i=\infty$, for every $\infty \in H$. The set $(S)=\left\{S+i: i \in Z_{u}\right\}$ is called the orbit of $S$ under $Z_{u}$ and $S$ is a base block of $(S)$.

To solve the Doyen-Wilson problem for 3-sun systems we use the difference method (see $[11,12])$. For every pair of distinct elements $i, j \in Z_{u}$, define $|i-j|_{u}=\min \{\mid i-$ $j|, u-| i-j \mid\}$ and set $D_{u}=\left\{|i-j|_{u}: i, j \in Z_{u}\right\}=\left\{1,2, \ldots,\left\lfloor\frac{u}{2}\right\rfloor\right\}$. The elements of $D_{u}$ are called differences of $Z_{u}$. For any $d \in D_{u}, d \neq \frac{u}{2}$, we can form a single 2factor $\left\{\{i, d+i\}: i \in Z_{u}\right\}$, while if $u$ is even and $d=\frac{u}{2}$, then we can form a 1-factor $\left\{\left\{i, i+\frac{u}{2}\right\}: 0 \leq i \leq \frac{u}{2}-1\right\}$. It is also worth remarking that 2-factors obtained from distinct differences are disjoint from each other and from the 1-factor.

If $D \subseteq D_{u}$, denote by $\left\langle Z_{u} \cup H, D\right\rangle$ the graph with vertex-set $V=Z_{u} \cup H$ and the edge-set $E=\left\{\{i, j\}:|i-j|_{u}=d, d \in D\right\} \cup\left\{\{\infty, i\}: \infty \in H, i \in Z_{u}\right\}$. The graph $\left\langle Z_{u} \cup H, D_{u}\right\rangle$ is the complete graph $K_{u+t} \backslash K_{t}$ based on $Z_{u} \cup H$ and having $H$ as a hole. The elements of $H$ are called infinity points.

Let $X$ be a set of size $n \equiv 0,1,4,9(\bmod 12)$. The aim of the paper is to decompose the graph $\left\langle Z_{u} \cup X, D_{u}\right\rangle$ into 3 -suns. To obtain our main result the $\left\langle Z_{u} \cup X, D_{u}\right\rangle$ will be regarded as a union of suitable edge-disjoint subgraphs of type $\left\langle Z_{u} \cup H, D\right\rangle$ (where $H \subseteq X$ may be empty, while $D \subseteq D_{u}$ is always non empty) and then each subgraph will be decomposed into 3 -suns by using the lemmas given in this section. From here on suppose $u \equiv 0,1,3,4,5,7,8,9,11(\bmod 12)$.

Lemmas 2.1-2.4 give decompositions of subgraphs of type $\left\langle Z_{u} \cup H, D\right\rangle$ where $D$ 
contains particular differences, more precisely, $D=\{2\}, D=\{2,4\}$ or $D=\left\{1, \frac{u}{3}\right\}$.

Lemma 2.1. Let $u \equiv 0(\bmod 4), u \geq 8$. Then the graph $\left\langle Z_{u} \cup\left\{\infty_{1}, \infty_{2}\right\},\{2\}\right\rangle$ can be decomposed into 3-suns.

Proof. Consider the 3 -suns

$$
\begin{aligned}
& \left(\infty_{1}, 2+4 i, 4 i ; 3+4 i, 4+4 i, \infty_{2}\right), \\
& \left(\infty_{2}, 3+4 i, 1+4 i ; 2+4 i, 5+4 i, \infty_{1}\right),
\end{aligned}
$$

for $i=0,1, \ldots, \frac{u}{4}-1$.

Lemma 2.2. Let $u \equiv 0(\bmod 12)$. Then the graph $\left\langle Z_{u} \cup\left\{\infty_{1}, \infty_{2}, \infty_{3}, \infty_{4}\right\},\{2\}\right\rangle$ can be decomposed into 3-suns.

Proof. Consider the 3 -suns

$$
\begin{aligned}
& \left(\infty_{1}, 12 i, 2+12 i ; 7+12 i,, \infty_{3}, \infty_{4}\right), \\
& \left(\infty_{1}, 4+12 i, 6+12 i ; 9+12 i, \infty_{3}, \infty_{4}\right), \\
& \left(\infty_{1}, 8+12 i, 10+12 i ; 11+12 i, \infty_{3}, \infty_{4}\right), \\
& \left(\infty_{2}, 2+12 i, 4+12 i ; 1+12 i, \infty_{3}, \infty_{4}\right), \\
& \left(\infty_{2}, 6+12 i, 8+12 i ; 7+12 i, \infty_{3}, \infty_{4}\right), \\
& \left(\infty_{2}, 10+12 i, 12+12 i ; 11+12 i, \infty_{3}, \infty_{4}\right), \\
& \left(\infty_{3}, 1+12 i, 3+12 i ; 9+12 i, \infty_{1}, \infty_{2}\right), \\
& \left(\infty_{3}, 5+12 i, 7+12 i ; 11+12 i, \infty_{1}, 9+12 i\right), \\
& \left(\infty_{4}, 3+12 i, 5+12 i ; 1+12 i, \infty_{1}, \infty_{2}\right), \\
& \left(\infty_{4}, 9+12 i, 11+12 i ; 7+12 i, \infty_{2}, 13+12 i\right),
\end{aligned}
$$

for $i=0,1, \ldots, \frac{u}{12}-1$.

Lemma 2.3. The graph $\left\langle Z_{u} \cup\left\{\infty_{1}, \infty_{2}, \infty_{3}, \infty_{4}\right\},\{2,4\}\right\rangle, u \geq 7, u \neq 8$, can be decomposed into 3-suns.

Proof. Let $u=4 k+r$, with $r=0,1,3$, and consider the 3 -suns

$$
\begin{aligned}
& \left(\infty_{1}, 4+4 i, 6+4 i ; 5+4 i, 8+4 i, \infty_{4}\right), \\
& \left(\infty_{2}, 5+4 i, 7+4 i ; 6+4 i, 9+4 i, \infty_{1}\right), \\
& \left(\infty_{3}, 6+4 i, 8+4 i ; 7+4 i, 10+4 i, \infty_{2}\right), \\
& \left(\infty_{4}, 7+4 i, 9+4 i ; 8+4 i, 11+4 i, \infty_{3}\right),
\end{aligned}
$$

for $i=0,1, \ldots, k-3, k \geq 3$, plus the following blocks as the case may be.

If $r=0$,

$$
\begin{aligned}
& \left(\infty_{1}, 0,2 ; 1,4, \infty_{4}\right), \\
& \left(\infty_{2}, 1,3 ; 2,5, \infty_{1}\right), \\
& \left(\infty_{3}, 2,4 ; 3,6, \infty_{2}\right), \\
& \left(\infty_{4}, 3,5 ; 4,7, \infty_{3}\right), \\
& \left(\infty_{1}, 4 k-4,4 k-2 ; 4 k-3,0, \infty_{4}\right),
\end{aligned}
$$




$$
\begin{aligned}
& \left(\infty_{2}, 4 k-3,4 k-1 ; 4 k-2,1, \infty_{1}\right), \\
& \left(\infty_{3}, 4 k-2,0 ; 4 k-1,2, \infty_{2}\right), \\
& \left(\infty_{4}, 4 k-1,1 ; 0,3, \infty_{3}\right)
\end{aligned}
$$

If $r=1$,

$$
\begin{aligned}
& \left(\infty_{1}, 0,2 ; 1,4, \infty_{2}\right), \\
& \left(\infty_{2}, 1,3 ; 0,5, \infty_{1}\right), \\
& \left(\infty_{3}, 2,4 ; 3,6, \infty_{2}\right), \\
& \left(\infty_{4}, 3,5 ; 4,7, \infty_{3}\right), \\
& \left(\infty_{1}, 4 k-4,4 k-2 ; 4 k-3,4 k, \infty_{2}\right), \\
& \left(\infty_{2}, 4 k-3,4 k-1 ; 4 k, 0, \infty_{1}\right), \\
& \left(\infty_{3}, 4 k-2,4 k ; 4 k-1,1, \infty_{1}\right), \\
& \left(\infty_{4}, 4 k-1,0 ; 4 k-2,2, \infty_{3}\right), \\
& \left(\infty_{4}, 4 k, 1 ; 2,3, \infty_{3}\right) .
\end{aligned}
$$

If $r=3$,

$$
\begin{aligned}
& \left(\infty_{1}, 0,2 ; 1,4, \infty_{4}\right), \\
& \left(\infty_{2}, 1,3 ; 2,5, \infty_{1}\right), \\
& \left(\infty_{3}, 2,4 ; 3,6, \infty_{2}\right), \\
& \left(\infty_{4}, 3,5 ; 4,7, \infty_{3}\right), \\
& \left(\infty_{1}, 4 k-4,4 k-2 ; 4 k-3,4 k, \infty_{4}\right), \\
& \left(\infty_{2}, 4 k-3,4 k-1 ; 4 k-2,4 k+1, \infty_{1}\right), \\
& \left(\infty_{3}, 4 k-2,4 k ; 4 k-1,4 k+2, \infty_{2}\right), \\
& \left(\infty_{4}, 4 k-1,4 k+1 ; 4 k, 0, \infty_{3}\right), \\
& \left(\infty_{1}, 4 k, 4 k+2 ; 4 k+1,1, \infty_{4}\right), \\
& \left(\infty_{2}, 4 k+1,0 ; 4 k+2,2, \infty_{4}\right), \\
& \left(\infty_{3}, 4 k+2,1 ; 0,3, \infty_{4}\right) .
\end{aligned}
$$

With regard to the difference 4 in $Z_{7}$, note that $|4|_{7}=3$ and the seven distinct blocks obtained for $k=1$ and $r=3$ gives a decomposition of $\left\langle Z_{7} \cup\left\{\infty_{1}, \infty_{2}, \infty_{3}, \infty_{4}\right\},\{2,3\}\right\rangle$ into 3 -suns.

Lemma 2.4. Let $u \equiv 0(\bmod 3), u \geq 12$. Then the graph $\left\langle Z_{u} \cup\left\{\infty_{1}, \infty_{2}, \ldots, \infty_{8}\right\}\right.$, $\left.\left\{1, \frac{u}{3}\right\}\right\rangle$ can be decomposed into 3-suns.

Proof. If $u \equiv 0(\bmod 6)$ consider the 3 -suns:

$$
\begin{aligned}
& \left(\infty_{1}, 2 i, \frac{u}{3}+2 i ; 2 \frac{u}{3}+2 i, \infty_{5}, \infty_{6}\right), i=0,1, \ldots, \frac{u}{6}-1, \\
& \left(\infty_{1}, 1+2 i, \frac{u}{3}+1+2 i ; 2 \frac{u}{3}+1+2 i, \infty_{6}, \infty_{5}\right), i=0,1, \ldots, \frac{u}{6}-1, \\
& \left(\infty_{2}, 2 \frac{u}{3}+2 i, \frac{u}{3}+2 i ; 2+2 i, 2 i, \infty_{5}\right), i=0,1, \ldots, \frac{u}{6}-2, \\
& \left(\infty_{2}, 2 \frac{u}{3}+1+2 i, \frac{u}{3}+1+2 i ; 3+2 i, 1+2 i, \infty_{6}\right), i=0,1, \ldots, \frac{u}{6}-2, \\
& \left(\infty_{2}, u-2,2 \frac{u}{3}-2 ; 0, \frac{u}{3}-2, \infty_{5}\right),
\end{aligned}
$$




$$
\begin{aligned}
& \left(\infty_{2}, u-1,2 \frac{u}{3}-1 ; 1, \frac{u}{3}-1, \infty_{6}\right), \\
& \left(\infty_{3}, 2 i, 1+2 i ; 2 \frac{u}{3}+2 i, \infty_{7}, \infty_{8}\right), i=0,1, \ldots, \frac{u}{6}-1, \\
& \left(\infty_{3}, \frac{u}{3}+2 i, \frac{u}{3}+1+2 i ; 2 \frac{u}{3}+1+2 i, \infty_{7}, \infty_{8}\right), i=0,1, \ldots, \frac{u}{6}-1, \\
& \left(\infty_{4}, 1+2 i, 2+2 i ; 2 \frac{u}{3}+2+2 i, \infty_{7}, \infty_{8}\right), i=0,1, \ldots, \frac{u}{6}-1, \\
& \left(\infty_{4}, \frac{u}{3}+1+2 i, \frac{u}{3}+2+2 i ; 2 \frac{u}{3}+1+2 i, \infty_{7}, \infty_{8}\right), i=0,1, \ldots, \frac{u}{6}-1, \\
& \left(\infty_{5}, 2 \frac{u}{3}+2 i, 2 \frac{u}{3}+1+2 i ; 1+2 i, \infty_{7}, \infty_{8}\right), i=0,1, \ldots, \frac{u}{6}-1, \\
& \left(\infty_{6}, 2 \frac{u}{3}+3+2 i, 2 \frac{u}{3}+4+2 i ; 2+2 i, \infty_{7}, \infty_{8}\right), i=0,1, \ldots, \frac{u}{6}-2, \\
& \left(\infty_{6}, 2 \frac{u}{3}+1,2 \frac{u}{3}+2 ; 2 \frac{u}{3}, \infty_{7}, \infty_{8}\right) .
\end{aligned}
$$

If $u \equiv 3(\bmod 6)$ consider the 3 -suns:

$$
\begin{aligned}
& \left(\infty_{1}, 2 i, \frac{u}{3}+2 i ; 2 \frac{u}{3}+2 i, \infty_{5}, \infty_{6}\right), i=0,1, \ldots, \frac{u-3}{6}, \\
& \left(\infty_{1}, 1+2 i, \frac{u}{3}+1+2 i ; 2 \frac{u}{3}+1+2 i, \infty_{6}, \infty_{5}\right), i=0,1, \ldots, \frac{u-9}{6}, \\
& \left(\infty_{2}, 2 \frac{u}{3}+2 i, \frac{u}{3}+2 i ; 2+2 i, 2 i, \infty_{5}\right), i=0,1, \ldots, \frac{u-9}{6}, \\
& \left(\infty_{2}, u-1,2 \frac{u}{3}-1 ; 0, \frac{u}{3}-1, \infty_{5}\right), \\
& \left(\infty_{2}, 2 \frac{u}{3}+1+2 i, \frac{u}{3}+1+2 i ; 3+2 i, 1+2 i, \infty_{6}\right), i=0,1, \ldots, \frac{u-15}{6}, \\
& \left(\infty_{2}, u-2,2 \frac{u}{3}-2 ; 1, \frac{u}{3}-2, \infty_{6}\right), \\
& \left(\infty_{3}, 2 i, 1+2 i ; 2 \frac{u}{3}+2 i, \infty_{7}, \infty_{8}\right), i=2,3, \ldots, \frac{u-3}{6}, \\
& \left(\infty_{3}, 0,1 ; 2 \frac{u}{3}, \infty_{6}, \infty_{8}\right), \\
& \left(\infty_{3}, 2,3 ; 2 \frac{u}{3}+2, \infty_{6}, \infty_{8}\right), \\
& \left(\infty_{3}, \frac{u}{3}+1+2 i, \frac{u}{3}+2+2 i ; 2 \frac{u}{3}+1+2 i, \infty_{7}, \infty_{8}\right), i=0,1, \ldots, \frac{u-9}{6}, \\
& \left(\infty_{4}, 1+2 i, 2+2 i ; 2 \frac{u}{3}+2+2 i, \infty_{7}, \infty_{8}\right), i=0,1, \ldots, \frac{u-9}{6}, \\
& \left(\infty_{4}, \frac{u}{3}+2 i, \frac{u}{3}+1+2 i ; 2 \frac{u}{3}+1+2 i, \infty_{7}, \infty_{8}\right), i=0,1, \ldots, \frac{u-3}{6}, \\
& \left(\infty_{5}, 2 \frac{u}{3}+2 i, 2 \frac{u}{3}+1+2 i ; 1+2 i, \infty_{7}, \infty_{8}\right), i=0,1, \ldots, \frac{u-9}{6}, \\
& \left(\infty_{6}, 2 \frac{u}{3}+1+2 i, 2 \frac{u}{3}+2+2 i ; 4+2 i, \infty_{7}, \infty_{8}\right), i=0,1, \ldots, \frac{u-15}{6}, \\
& \left(\infty_{6}, u-2, u-1 ; 2 \frac{u}{3}, \infty_{7}, \infty_{8}\right), \\
& \left(\infty_{7}, u-1,0 ; 2, \infty_{5}, \infty_{8}\right) .
\end{aligned}
$$

Lemmas $2.5-2.9$ allow to decompose $\left\langle Z_{u} \cup H, D\right\rangle$ where $u$ is even and $D$ contains the difference $\frac{u}{2}$.

Lemma 2.5. Let $u$ be even, $u \geq 8$. Then the graph $\left\langle Z_{u} \cup\left\{\infty_{1}, \infty_{2}, \infty_{3}\right\},\left\{1, \frac{u}{2}\right\}\right\rangle$ can be decomposed into 3-suns.

Proof. Consider the 3-suns

$$
\begin{aligned}
& \left(\infty_{1}, 2 i, 1+2 i ; \frac{u}{2}+2+2 i, \frac{u}{2}+2 i, \infty_{3}\right), i=0,1, \ldots, \frac{u}{4}-2, \\
& \left(\infty_{1}, \frac{u}{2}-2, \frac{u}{2}-1 ; \frac{u}{2}, u-2, \infty_{3}\right) \\
& \left(\infty_{2}, 1+2 i, \frac{u}{2}+1+2 i ; 2 i, 2+2 i, \infty_{1}\right), i=0,1, \ldots, \frac{u}{4}-1, \\
& \left(\infty_{3}, \frac{u}{2}+1+2 i, \frac{u}{2}+2 i ; 2 i, \frac{u}{2}+2+2 i, \infty_{2}\right), i=0,1, \ldots, \frac{u}{4}-1 .
\end{aligned}
$$

Lemma 2.6. Let $u \equiv 0(\bmod 12)$. Then the graph $\left\langle Z_{u} \cup\left\{\infty_{1}, \infty_{2}, \infty_{3}, \infty_{4}\right\},\left\{1, \frac{u}{2}\right\}\right\rangle$ can be decomposed into 3-suns. 
Proof. Consider the 3 -suns

$$
\begin{aligned}
& \left(\infty_{1}, 6 i, \frac{u}{2}+6 i ; 4+6 i, \infty_{3}, \infty_{2}\right), \\
& \left(\infty_{1}, 1+6 i, \frac{u}{2}+1+6 i ; 5+6 i, \infty_{4}, \infty_{2}\right), \\
& \left(\infty_{1}, 2+6 i, \frac{u}{2}+2+6 i ; \frac{u}{2}+3+6 i, \infty_{4}, \infty_{3}\right), \\
& \left(\infty_{2}, 1+6 i, 6 i ; \frac{u}{2}+3+6 i, \infty_{3}, \infty_{4}\right), \\
& \left(\infty_{2}, 2+6 i, 3+6 i ; \frac{u}{2}+4+6 i, 1+6 i, \infty_{4}\right), \\
& \left(\infty_{2}, 5+6 i, 4+6 i ; \frac{u}{2}+5+6 i, 6+6 i, 3+6 i\right), \\
& \left(\infty_{3}, 3+6 i, \frac{u}{2}+3+6 i ; 2+6 i, \infty_{1}, \frac{u}{2}+2+6 i\right), \\
& \left(\infty_{3}, 4+6 i, \frac{u}{2}+4+6 i ; \frac{u}{2}+6 i, \infty_{4}, \infty_{1}\right), \\
& \left(\infty_{3}, 5+6 i, \frac{u}{2}+5+6 i ; \frac{u}{2}+1+6 i, \infty_{4}, \infty_{1}\right), \\
& \left(\infty_{4}, \frac{u}{2}+1+6 i, \frac{u}{2}+2+6 i ; \frac{u}{2}+3+6 i, \frac{u}{2}+6 i, \infty_{2}\right), \\
& \left(\infty_{4}, \frac{u}{2}+4+6 i, \frac{u}{2}+5+6 i ; \frac{u}{2}+6 i, \frac{u}{2}+3+6 i, \frac{u}{2}+6+6 i\right),
\end{aligned}
$$

for $i=0,1, \ldots, \frac{u}{12}-1$.

Lemma 2.7. Let $u$ be even, $u \geq 8$. Then the graph $\left\langle Z_{u} \cup\left\{\infty_{1}, \infty_{2}, \ldots, \infty_{6}\right\},\left\{1, \frac{u}{2}\right\}\right\rangle$ can be decomposed into 3-suns.

Proof. Consider the 3 -suns

$$
\begin{aligned}
& \left(\infty_{1}, 2 i, 1+2 i ; \frac{u}{2}+2+2 i, \frac{u}{2}+2 i, \infty_{3}\right), i=0,1, \ldots, \frac{u}{4}-2, \\
& \left(\infty_{1}, \frac{u}{2}-2, \frac{u}{2}-1 ; \frac{u}{2}, u-2, \infty_{3}\right), \\
& \left(\infty_{2}, 1+2 i, \frac{u}{2}+1+2 i ; 2 i, \infty_{6}, \infty_{1}\right), i=0,1, \ldots, \frac{u}{4}-1, \\
& \left(\infty_{3}, \frac{u}{2}+1+2 i, \frac{u}{2}+2 i ; 2 i, \infty_{6}, \infty_{2}\right), i=0,1, \ldots, \frac{u}{4}-1, \\
& \left(\infty_{4}, 1+2 i, 2+2 i ; \frac{u}{2}+2+2 i, \infty_{5}, \infty_{6}\right), i=0,1, \ldots, \frac{u}{4}-1, \\
& \left(\infty_{5}, \frac{u}{2}+1+2 i, \frac{u}{2}+2+2 i ; 2+2 i, \infty_{4}, \infty_{6}\right), i=0,1, \ldots, \frac{u}{4}-1 .
\end{aligned}
$$

Lemma 2.8. Let $u \equiv 0(\bmod 12)$. Then the graph $\left\langle Z_{u} \cup\left\{\infty_{1}, \infty_{2}, \ldots, \infty_{7}\right\},\left\{1, \frac{u}{2}\right\}\right\rangle$ can be decomposed into 3-suns.

Proof. Consider the 3 -suns

$$
\begin{aligned}
& \left(\infty_{1}, 6 i, \frac{u}{2}+6 i ; 4+6 i, \infty_{7}, \infty_{2}\right), \\
& \left(\infty_{1}, 1+6 i, \frac{u}{2}+1+6 i ; \frac{u}{2}+3+6 i, \infty_{7}, \infty_{4}\right), \\
& \left(\infty_{1}, 2+6 i, \frac{u}{2}+2+6 i ; \frac{u}{2}+5+6 i, \infty_{5}, \infty_{2}\right), \\
& \left(\infty_{2}, 3+6 i, \frac{u}{2}+3+6 i ; 6 i, \infty_{1}, \infty_{4}\right), \\
& \left(\infty_{2}, 4+6 i, \frac{u}{2}+4+6 i ; 2+6 i, \infty_{7}, \infty_{1}\right), \\
& \left(\infty_{2}, 5+6 i, \frac{u}{2}+5+6 i ; \frac{u}{2}+1+6 i, \infty_{1}, \infty_{7}\right), \\
& \left(\infty_{3}, 6 i, 1+6 i ; \frac{u}{2}+6 i, \infty_{5}, \infty_{6}\right), \\
& \left(\infty_{3}, 2+6 i, 3+6 i ; \frac{u}{2}+2+6 i, \infty_{7}, \infty_{6}\right), \\
& \left(\infty_{3}, 4+6 i, 5+6 i ; \frac{u}{2}+5+6 i, \infty_{5}, \infty_{6}\right), \\
& \left(\infty_{4}, 1+6 i, 2+6 i ; \frac{u}{2}+6+6 i, \infty_{2}, \infty_{6}\right), \\
& \left(\infty_{4}, 3+6 i, 4+6 i ; \frac{u}{2}+4+6 i, \infty_{7}, \infty_{6}\right),
\end{aligned}
$$




$$
\begin{aligned}
& \left(\infty_{4}, 5+6 i, 6+6 i ; \frac{u}{2}+5+6 i, \infty_{7}, \infty_{6}\right), \\
& \left(\infty_{5}, \frac{u}{2}+6 i, \frac{u}{2}+1+6 i ; 1+6 i, \infty_{7}, \infty_{3}\right), \\
& \left(\infty_{5}, \frac{u}{2}+2+6 i, \frac{u}{2}+3+6 i ; 3+6 i, \infty_{7}, \infty_{3}\right), \\
& \left(\infty_{5}, \frac{u}{2}+4+6 i, \frac{u}{2}+5+6 i ; 5+6 i, \infty_{7}, \frac{u}{2}+6+6 i\right), \\
& \left(\infty_{6}, \frac{u}{2}+1+6 i, \frac{u}{2}+2+6 i ; \frac{u}{2}+5+6 i, \infty_{7}, \infty_{4}\right), \\
& \left(\infty_{6}, \frac{u}{2}+3+6 i, \frac{u}{2}+4+6 i ; \frac{u}{2}+6+6 i, \infty_{7}, \infty_{3}\right),
\end{aligned}
$$

for $i=0,1, \ldots, \frac{u}{12}-1$.

Lemma 2.9. Let $u \equiv 0(\bmod 12)$. Then the graph $\left\langle Z_{u} \cup\left\{\infty_{1}, \infty_{2}, \infty_{3}\right\},\left\{1,2, \frac{u}{2}\right\}\right\rangle$ can be decomposed into 3 -suns.

Proof. Consider the 3 -suns

$$
\begin{aligned}
& \left(\infty_{1}, 6 i, 1+6 i ; \frac{u}{2}+1+6 i, \frac{u}{2}+6 i, 3+6 i\right), \\
& \left(\infty_{1}, 2+6 i, 3+6 i ; \frac{u}{2}+5+6 i, \frac{u}{2}+2+6 i, 5+6 i\right), \\
& \left(\infty_{1}, 4+6 i, 5+6 i ; \frac{u}{2}+2+6 i, \frac{u}{2}+4+6 i, 7+6 i\right), \\
& \left(\infty_{1}, \frac{u}{2}+3+6 i, \frac{u}{2}+4+6 i ; \frac{u}{2}+6 i, \frac{u}{2}+2+6 i, \infty_{2}\right), \\
& \left(\infty_{2}, 1+6 i, \frac{u}{2}+1+6 i ; \frac{u}{2}+3+6 i, 2+6 i, \frac{u}{2}+2+6 i\right), \\
& \left(\infty_{2}, 3+6 i, 4+6 i ; 2+6 i, \frac{u}{2}+3+6 i, 6+6 i\right), \\
& \left(\infty_{2}, 5+6 i, \frac{u}{2}+5+6 i ; \frac{u}{2}+2+6 i, 6+6 i, \frac{u}{2}+6+6 i\right), \\
& \left(\infty_{3}, 2+6 i, 6 i ; 1+6 i, 4+6 i, \infty_{2}\right), \\
& \left(\infty_{3}, \frac{u}{2}+2+6 i, \frac{u}{2}+6 i ; 4+6 i, \frac{u}{2}+4+6 i, \infty_{2}\right), \\
& \left(\infty_{3}, \frac{u}{2}+1+6 i, \frac{u}{2}+3+6 i ; 3+6 i, \frac{u}{2}+6 i, \frac{u}{2}+5+6 i\right), \\
& \left(\infty_{3}, \frac{u}{2}+5+6 i, \frac{u}{2}+4+6 i ; 5+6 i, \frac{u}{2}+7+6 i, \frac{u}{2}+6+6 i\right),
\end{aligned}
$$

for $i=0,1, \ldots, \frac{u}{12}-1$.

The following lemma "combines" one infinity point with one difference $d \neq \frac{u}{2}, \frac{u}{3}$ such that $\frac{u}{\operatorname{gcd}(u, d)} \equiv 0(\bmod 3)($ therefore, $u \equiv 0(\bmod 3))$.

Lemma 2.10. Let $u \equiv 0(\bmod 3)$ and $d \in D_{u} \backslash\left\{\frac{u}{2}, \frac{u}{3}\right\}$ such that $p=\frac{u}{\operatorname{gcd}(u, d)} \equiv 0$ (mod 3). Then the graph $\left\langle Z_{u} \cup\{\infty\},\{d\}\right\rangle$ can be decomposed into 3-suns.

Proof. The subgraph $\left\langle Z_{u},\{d\}\right\rangle$ can be decomposed into $\frac{u}{p}$ cycles of length $p=3 q, q \geq 2$.

If $q>2$, let $\left(x_{1}, x_{2}, \ldots, x_{3 q}\right)$ be a such cycle and consider the 3 -suns

$$
\left(\infty, x_{2+3 i}, x_{3+3 i} ; x_{7+3 i}, x_{1+3 i}, x_{4+3 i}\right),
$$

for $i=0,1, \ldots, q-1$ (where the sum is modulo $3 q$ ).

If $q=2$, let $\left(x_{1}^{(j)}, x_{2}^{(j)}, x_{3}^{(j)}, x_{4}^{(j)}, x_{5}^{(j)}, x_{6}^{(j)}\right), j=0,1, \ldots, \frac{u}{6}-1$, be the 6 -cycles decomposing $\left\langle Z_{u},\{d\}\right\rangle$ and consider the 3 -suns

$$
\begin{aligned}
& \left(\infty, x_{2}^{(j)}, x_{3}^{(j)} ; x_{1}^{(j+1)}, x_{1}^{(j)}, x_{4}^{(j)}\right), \\
& \left(\infty, x_{5}^{(j)}, x_{6}^{(j)} ; x_{4}^{(j+1)}, x_{4}^{(j)}, x_{1}^{(j)}\right),
\end{aligned}
$$

for $j=0,1, \ldots, \frac{u}{6}-1$ (where the sums are modulo $\frac{u}{6}$ ). 
Subsequent Lemmas $2.11-2.14$ allow to decompose $\left\langle Z_{u} \cup H, D\right\rangle$, where $|H|=1,2$, $3,5,|D|=6-|H|$ and $\frac{u}{2} \notin D$; here, $u$ and $D$ are any with the unique condition that if $D$ contains at least three differences $d_{1}, d_{2}, d_{3}$, then $d_{3}=d_{2}-d_{1}$ or $d_{1}+d_{2}+d_{3}=u$.

Lemma 2.11. Let $d_{1}, d_{2}, d_{3}, d_{4}, d_{5} \in D_{u} \backslash\left\{\frac{u}{2}\right\}$ such that $d_{3}=d_{2}-d_{1}$ or $d_{1}+d_{2}+d_{3}=u$. Then the graph $\left\langle Z_{u} \cup\{\infty\},\left\{d_{1}, d_{2}, d_{3}, d_{4}, d_{5}\right\}\right\rangle$ can be decomposed into 3-suns.

Proof. If $d_{3}=d_{2}-d_{1}$, consider the orbit of

$$
\left(d_{1}, d_{2}, 0 ; \infty, d_{2}+d_{5}, d_{4}\right)
$$

(or $\left(d_{1}, d_{2}, 0 ; \infty, d_{2}+d_{5},-d_{4}\right)$, if $\left.d_{2}+d_{5}=d_{4}\right)$ under $Z_{u}$. If $d_{1}+d_{2}+d_{3}=u$, consider the orbit of

$$
\left(-d_{1}, d_{2}, 0 ; \infty, d_{2}+d_{5}, d_{4}\right)
$$

(or $\left(-d_{1}, d_{2}, 0 ; \infty, d_{2}+d_{5},-d_{4}\right)$, if $d_{2}+d_{5}=d_{4}$ ) under $Z_{u}$.

Lemma 2.12. Let $d_{1}, d_{2}, d_{3}, d_{4} \in D_{u} \backslash\left\{\frac{u}{2}\right\}$ such that $d_{3}=d_{2}-d_{1}$ or $d_{1}+d_{2}+d_{3}=u$. Then the graph $\left\langle Z_{u} \cup\left\{\infty_{1}, \infty_{2}\right\},\left\{d_{1}, d_{2}, d_{3}, d_{4}\right\}\right\rangle$ can be decomposed into 3 -suns.

Proof. Consider the orbit of $\left(d_{1}, d_{2}, 0 ; \infty_{1}, \infty_{2}, d_{4}\right)$ or $\left(-d_{1}, d_{2}, 0 ; \infty_{1}, \infty_{2}, d_{4}\right)$ under $Z_{u}$ when, respectively, $d_{3}=d_{2}-d_{1}$ or $d_{1}+d_{2}+d_{3}=u$.

Lemma 2.13. Let $d_{1}, d_{2}, d_{3} \in D_{u} \backslash\left\{\frac{u}{2}\right\}$ such that $d_{3}=d_{2}-d_{1}$ or $d_{1}+d_{2}+d_{3}=u$. Then the graph $\left\langle Z_{u} \cup\left\{\infty_{1}, \infty_{2}, \infty_{3}\right\},\left\{d_{1}, d_{2}, d_{3}\right\}\right\rangle$ can be decomposed into 3-suns.

Proof. Consider the orbit of $\left(d_{1}, d_{2}, 0 ; \infty_{1}, \infty_{2}, \infty_{3}\right)$ or $\left(-d_{1}, d_{2}, 0 ; \infty_{1}, \infty_{2}, \infty_{3}\right)$ under $Z_{u}$ when, respectively, $d_{3}=d_{2}-d_{1}$ or $d_{1}+d_{2}+d_{3}=u$.

Lemma 2.14. Let $d \in D_{u} \backslash\left\{\frac{u}{2}\right\}$, the graph $\left\langle Z_{u} \cup\left\{\infty_{1}, \infty_{2}, \infty_{3}, \infty_{4}, \infty_{5}\right\},\{d\}\right\rangle$ can be decomposed into 3-suns.

Proof. The subgraph $\left\langle Z_{u},\{d\}\right\rangle$ is regular of degree 2 and so can be decomposed into $l$ cycles, $l \geq 3$. Let $\left(x_{1}, x_{2}, \ldots, x_{l}\right)$ be a such cycle. Put $l=3 q+r$, with $r=0,1,2$, and consider the 3 -suns with the sums modulo $l$

$$
\begin{aligned}
& \left(\infty_{1}, x_{1+3 i}, x_{2+3 i} ; x_{3+3 i}, \infty_{4}, \infty_{5}\right), \\
& \left(\infty_{2}, x_{2+3 i}, x_{3+3 i} ; x_{4+3 i}, \infty_{4}, \infty_{5}\right), \\
& \left(\infty_{3}, x_{3+3 i}, x_{4+3 i} ; x_{5+3 i}, \infty_{4}, \infty_{5}\right),
\end{aligned}
$$

for $i=0,1, \ldots, q-2, q \geq 2$, plus the following blocks as the case may be.

If $r=0$,

$$
\begin{aligned}
& \left(\infty_{1}, x_{3 q-2}, x_{3 q-1} ; x_{3 q}, \infty_{4}, \infty_{5}\right) \\
& \left(\infty_{2}, x_{3 q-1}, x_{3 q} ; x_{1}, \infty_{4}, \infty_{5}\right) \\
& \left(\infty_{3}, x_{3 q}, x_{1} ; x_{2}, \infty_{4}, \infty_{5}\right)
\end{aligned}
$$

If $r=1$,

$$
\begin{aligned}
& \left(\infty_{1}, x_{3 q-2}, x_{3 q-1} ; x_{3 q+1}, \infty_{4}, \infty_{5}\right), \\
& \left(\infty_{2}, x_{3 q-1}, x_{3 q} ; x_{1}, \infty_{4}, \infty_{1}\right) \\
& \left(\infty_{3}, x_{3 q}, x_{3 q+1} ; x_{2}, \infty_{4}, \infty_{2}\right) \\
& \left(\infty_{5}, x_{3 q+1}, x_{1} ; x_{3 q}, \infty_{4}, \infty_{3}\right) .
\end{aligned}
$$


If $r=2$,

$$
\begin{aligned}
& \left(\infty_{1}, x_{3 q-2}, x_{3 q-1} ; x_{3 q+2}, \infty_{4}, \infty_{5}\right), \\
& \left(\infty_{2}, x_{3 q-1}, x_{3 q} ; x_{1}, \infty_{4}, \infty_{5}\right), \\
& \left(\infty_{3}, x_{3 q}, x_{3 q+1} ; x_{2}, \infty_{1}, \infty_{2}\right), \\
& \left(\infty_{4}, x_{3 q+1}, x_{3 q+2} ; x_{3 q}, \infty_{1}, \infty_{3}\right), \\
& \left(\infty_{5}, x_{3 q+2}, x_{1} ; x_{3 q+1}, \infty_{2}, \infty_{3}\right) .
\end{aligned}
$$

Finally, after settling the infinity points by using the above lemmas, if $u$ is large we need to decompose the subgraph $\left\langle Z_{u}, L\right\rangle$, where $L$ is the set of the differences unused (difference leave). Since by applying Lemmas $2.1-2.13$ it could be necessary to use the differences 1 , 2 or 4 , while Lemma 2.14 does not impose any restriction, it is possible to combine infinity points and differences in such a way that the difference leave $L$ is the set of the "small" differences, where 1, 2 or 4 could possibly be avoided.

Lemma 2.15. Let $\alpha \in\{0,4,8\}$ and $u$, $s$ be positive integers such that $u>12 s+\alpha$. Then there exists a decomposition of $\left\langle Z_{u}, L\right\rangle$ into 3 -suns, where:

i) $\alpha=0$ and $L=[1,6 s]$;

ii) $\alpha=4$ and $L=[3,6 s+2]$;

iii) $\alpha=8$ and $L=[3,6 s+4] \backslash\{4,6 s+3\}$.

Proof.

i) Consider the orbits $\left(S_{j}\right)$ under $Z_{u}$, where $S_{j}=(5 s+1+j, 5 s-j, 0 ; 3 s, s, u-$ $2-2 j), j=0,1, \ldots, s-1$.

ii) Consider the orbits in i), where $\left(S_{0}\right)$ is replaced with the orbit of $(6 s+1,4 s, 0 ; s, 9 s$, $6 s+2)$.

iii) Consider the orbits in i), where the orbits $\left(S_{0}\right)$ and $\left(S_{1}\right)$ are replaced with the orbits of $(6 s+1,4 s, 0 ; s, 9 s, 6 s+4)$ and $(5 s+2,5 s-1,0 ; 3 s, s, 6 s+2)$.

\section{The main result}

Let $(X, \mathcal{S})$ be a $3 \mathrm{SS}(n)$ and $m \equiv 0,1,4,9(\bmod 12)$.

Lemma 3.1. If $(X, \mathcal{S})$ is embedded in a 3-sun system of order $m>n$, then $m \geq \frac{7}{5} n+1$.

Proof. Suppose $(X, \mathcal{S})$ is embedded in $\left(X^{\prime}, \mathcal{S}^{\prime}\right)$, with $\left|X^{\prime}\right|=m=n+u(u$ positive integer). Let $c_{i}$ be the number of 3 -suns of $\mathcal{S}^{\prime}$ each of which contains exactly $i$ edges in $X^{\prime} \backslash X$. Then $\sum_{i=1}^{6} i \times c_{i}=\left(\begin{array}{l}u \\ 2\end{array}\right)$ and $\sum_{i=1}^{5}(6-i) c_{i}=u \times n$, from which it follows $6 c_{2}+12 c_{3}+18 c_{4}+24 c_{5}+30 c_{6}=\frac{u(5 u-2 n-5)}{2}$ and so $u \geq \frac{2}{5} n+1$ and $m \geq \frac{7}{5} n+1$.

By previous Lemma:

1. if $n=60 k+5 r, r=0,5,8,9$, then $m \geq 84 k+7 r+1$;

2. if $n=60 k+5 r+1, r=0,3,4,7$, then $m \geq 84 k+7 r+3$;

3. if $n=60 k+5 r+2, r=2,7,10,11$, then $m \geq 84 k+7 r+4$;

4. if $n=60 k+5 r+3, r=2,5,6,9$, then $m \geq 84 k+7 r+6$; 
5. if $n=60 k+5 r+4, r=0,1,4,9$, then $m \geq 84 k+7 r+7$.

In order to prove that the necessary conditions for embedding a 3-sun system $(X, \mathcal{S})$ of order $n$ in a 3 -sun system of order $m=n+u, u>0$ are also sufficient, the graph $\left\langle Z_{u} \cup X, D_{u}\right\rangle$ will be expressed as a union of edge-disjoint subgraphs $\left\langle Z_{u} \cup X, D_{u}\right\rangle=$ $\left\langle Z_{u} \cup X, D\right\rangle \cup\left\langle Z_{u}, L\right\rangle$, where $L=D_{u} \backslash D$ is the difference leave, and $\left\langle Z_{u} \cup X, D\right\rangle$ (if necessary, expressed itself as a union of subgraphs) will be decomposed by using Lemmas 2.1-2.14, while if $L \neq \emptyset,\left\langle Z_{u}, L\right\rangle$ will be decomposed by Lemma 2.15. To obtain our main result we will distinguish the five cases $1 .-5$. listed before by giving a general proof for any $k \geq 0$ with the exception of a few cases for $k=0$, which will be indicated by a star $\star$ and solved in Appendix. Finally, note that:
a) $u \equiv 0,1,4$, or $9(\bmod 12)$, if $n \equiv 0(\bmod 12)$;
b) $u \equiv 0,3,8$, or $11(\bmod 12)$, if $n \equiv 1(\bmod 12)$;
c) $u \equiv 0,5,8$, or $9(\bmod 12), n \equiv 4(\bmod 12)$;
d) $u \equiv 0,3,4$, or $7(\bmod 12)$, if $n \equiv 9(\bmod 12)$.

Proposition 3.2. For any $n=60 k+5 r, r=0,5,8,9$, there exists a decomposition of $K_{n+u} \backslash K_{n}$ into 3 -suns for every admissible $u \geq 24 k+2 r+1$.

Proof. Let $X=\left\{\infty_{1}, \infty_{2}, \ldots, \infty_{60 k+5 r}\right\}, r=0,5,8,9$, and $u=24 k+2 r+1+h$, with $h \geq 0$. Set $h=12 s+l, 0 \leq l \leq 11$ ( $l$ depends on $r$ ), and distinguish the following cases.

Case 1: $r=0,5,8,9$ and $l=0(\operatorname{odd} u)$.

Write $\left\langle Z_{u} \cup X, D_{u}\right\rangle=\left\langle Z_{u} \cup X, D\right\rangle \cup\left\langle Z_{u}, L\right\rangle$, where $D=[6 s+1,12 k+r+6 s]$, $|D|=12 k+r$, and $L=[1,6 s]$, and apply Lemmas 2.14 and 2.15.

Case 2: $r=0,9$ and $l=8($ odd $u)$.

Write

$$
\begin{aligned}
&\left\langle Z_{u} \cup X, D_{u}\right\rangle=\langle\left\langle Z_{u} \cup\left\{\infty_{1}, \infty_{2}, \infty_{3}\right\},\{2,6 s+3,6 s+5\}\right\rangle \cup \\
&\left\langle Z_{u} \cup\left\{\infty_{4}\right\},\{1\}\right\rangle \cup\left\langle Z_{u} \cup\left\{\infty_{5}\right\},\{6 s+4\}\right\rangle \cup \\
&\left\langle Z_{u} \cup\left(X \backslash\left\{\infty_{1}, \infty_{2}, \infty_{3}, \infty_{4}, \infty_{5}\right\}\right), D^{\prime}\right\rangle \cup\left\langle Z_{u}, L\right\rangle,
\end{aligned}
$$

where $D^{\prime}=[6 s+6,12 k+r+6 s+4],\left|D^{\prime}\right|=12 k+r-1$, and $L=[3,6 s+2]$, and apply Lemmas 2.13, 2.10, 2.14 and 2.15.

Case 3: $r=5,8$ and $l=4($ odd $u)$.

Write

$$
\begin{aligned}
\left\langle Z_{u} \cup X, D_{u}\right\rangle=\left\langle Z_{u} \cup\left\{\infty_{1}, \infty_{2}, \infty_{3}, \infty_{4}\right\},\{2,4\}\right\rangle \cup\left\langle Z_{u} \cup\left\{\infty_{5}\right\},\{1\}\right\rangle \cup \\
\left\langle Z_{u} \cup\left(X \backslash\left\{\infty_{1}, \infty_{2}, \infty_{3}, \infty_{4}, \infty_{5}\right\}\right), D^{\prime}\right\rangle \cup\left\langle Z_{u}, L\right\rangle,
\end{aligned}
$$

where $D^{\prime}=[6 s+3,12 k+r+6 s+2] \backslash\{6 s+4\},\left|D^{\prime}\right|=12 k+r-1$, and $L=$ $[3,6 s+4] \backslash\{4,6 s+3\}$, and apply Lemmas 2.3, 2.10, 2.14 and 2.15.

Case 4: $r=0,8$ and $l=3$ (even $u$ ).

Write

$$
\begin{aligned}
\left\langle Z_{u} \cup X, D_{u}\right\rangle=\left\langle Z_{u} \cup\left\{\infty_{1}, \infty_{2}, \infty_{3}\right\},\left\{1, \frac{u}{2}\right\}\right\rangle \cup\left\langle Z_{u} \cup\left\{\infty_{4}, \infty_{5}\right\},\{2\}\right\rangle \cup \\
\left\langle Z_{u} \cup\left(X \backslash\left\{\infty_{1}, \infty_{2}, \infty_{3}, \infty_{4}, \infty_{5}\right\}\right), D^{\prime}\right\rangle \cup\left\langle Z_{u}, L\right\rangle,
\end{aligned}
$$


where $D^{\prime}=[6 s+3,12 k+r+6 s+1],\left|D^{\prime}\right|=12 k+r-1$, and $L=[3,6 s+2]$, and apply Lemmas 2.5, 2.1, 2.14 and 2.15.

Case 5: $r=0$ and $l=11$ (even $u$ ).

Write

$$
\begin{aligned}
\left\langle Z_{u} \cup X, D_{u}\right\rangle= & \left\langle Z_{u} \cup\left\{\infty_{1}, \infty_{2}, \infty_{3}\right\},\left\{1,2, \frac{u}{2}\right\}\right\rangle \cup \\
& \left\langle Z_{u} \cup\left\{\infty_{4}, \infty_{5}\right\},\{4,6 s+3,6 s+5,6 s+7\}\right\rangle \cup \\
& \left\langle Z_{u} \cup\left(X \backslash\left\{\infty_{1}, \infty_{2}, \infty_{3}, \infty_{4}, \infty_{5}\right\}\right), D^{\prime}\right\rangle \cup\left\langle Z_{u}, L\right\rangle,
\end{aligned}
$$

where $D^{\prime}=[6 s+6,12 k+6 s+5] \backslash\{6 s+7\},\left|D^{\prime}\right|=12 k-1$, and $L=[3,6 s+4] \backslash$ $\{4,6 s+3\}$, and apply Lemmas $2.9,2.12,2.14$ and 2.15 .

Case 6: $r=5$ and $l=1$ (even $u$ ).

Write

$$
\begin{aligned}
\left\langle Z_{u} \cup X, D_{u}\right\rangle=\left\langle Z_{u} \cup\left\{\infty_{1}, \infty_{2}, \ldots, \infty_{6}\right\},\left\{1, \frac{u}{2}\right\}\right\rangle \cup \\
\left\langle Z_{u} \cup\left\{\infty_{7}, \infty_{8}, \infty_{9}, \infty_{10}\right\},\{2\}\right\rangle \cup \\
\quad\left\langle Z_{u} \cup\left(X \backslash\left\{\infty_{1}, \infty_{2}, \ldots, \infty_{10}\right\}\right), D^{\prime}\right\rangle \cup\left\langle Z_{u}, L\right\rangle,
\end{aligned}
$$

where $D^{\prime}=[6 s+3,12 k+6 s+5],\left|D^{\prime}\right|=12 k+3$, and $L=[3,6 s+2]$, and apply Lemmas 2.7, 2.2, 2.14 and 2.15.

Case 7: $r=5,9$ and $l=9$ (even $u)$.

Write

$$
\begin{aligned}
\left\langle Z_{u} \cup X, D_{u}\right\rangle= & \left\langle Z_{u} \cup\left\{\infty_{1}, \infty_{2}, \infty_{3}\right\},\left\{1, \frac{u}{2}\right\}\right\rangle \cup \\
& \left\langle Z_{u} \cup\left\{\infty_{4}, \infty_{5}\right\},\{2,6 s+3,6 s+4,6 s+5\}\right\rangle \cup \\
& \left\langle Z_{u} \cup\left(X \backslash\left\{\infty_{1}, \infty_{2}, \infty_{3}, \infty_{4}, \infty_{5}\right\}\right), D^{\prime}\right\rangle \cup\left\langle Z_{u}, L\right\rangle,
\end{aligned}
$$

where $D^{\prime}=[6 s+6,12 k+r+6 s+4],\left|D^{\prime}\right|=12 k+r-1$, and $L=[3,6 s+2]$, and apply Lemmas 2.5, 2.12, 2.14 and 2.15.

Case 8: $r=8$ and $l=7$ (even $u$ ).

Write

$$
\begin{aligned}
\left\langle Z_{u} \cup X, D_{u}\right\rangle=\langle & \left\langle Z_{u} \cup\left\{\infty_{1}, \infty_{2}, \infty_{3}\right\},\left\{1,2, \frac{u}{2}\right\}\right\rangle \cup \\
& \left\langle Z_{u} \cup\left\{\infty_{4}\right\},\{4\}\right\rangle \cup\left\langle Z_{u} \cup\left\{\infty_{5}\right\},\{6 s+5\}\right\rangle \cup \\
& \left\langle Z_{u} \cup\left(X \backslash\left\{\infty_{1}, \infty_{2}, \infty_{3}, \infty_{4}, \infty_{5}\right\}\right), D^{\prime}\right\rangle \cup\left\langle Z_{u}, L\right\rangle,
\end{aligned}
$$

where $D^{\prime}=[6 s+3,12 k+6 s+11] \backslash\{6 s+4,6 s+5\},\left|D^{\prime}\right|=12 k+7$, and $L=$ $[3,6 s+4] \backslash\{4,6 s+3\}$, and apply Lemmas 2.9, 2.10, 2.14 and 2.15.

Case 9: $r=9$ and $l=5$ (even $u$ ).

Write

$$
\begin{aligned}
& \left\langle Z_{u} \cup X, D_{u}\right\rangle=\left\langle Z_{u} \cup\left\{\infty_{1}, \infty_{2}, \infty_{3}\right\},\left\{1, \frac{u}{2}\right\}\right\rangle \cup\left\langle Z_{u} \cup\left\{\infty_{4}\right\},\{2\}\right\rangle \cup \\
& \left\langle Z_{u} \cup\left\{\infty_{5}\right\},\{4\}\right\rangle \cup\left\langle Z_{u} \cup\left(X \backslash\left\{\infty_{1}, \infty_{2}, \infty_{3}, \infty_{4}, \infty_{5}\right\}\right), D^{\prime}\right\rangle \cup\left\langle Z_{u}, L\right\rangle,
\end{aligned}
$$

where $D^{\prime}=[6 s+3,12 k+6 s+11] \backslash\{6 s+4\},\left|D^{\prime}\right|=12 k+8$, and $L=[3,6 s+4] \backslash$ $\{4,6 s+3\}$, and apply Lemmas $2.5,2.10,2.14$ and 2.15 . 
Proposition 3.3. For any $n=60 k+5 r+1, r=0,3,4,7$, there exists a decomposition of $K_{n+u} \backslash K_{n}$ into 3 -suns for every admissible $u \geq 24 k+2 r+2$.

Proof. Let $X=\left\{\infty_{1}, \infty_{2}, \ldots, \infty_{60 k+5 r+1}\right\}, r=0,3,4,7$, and $u=24 k+2 r+2+h$, with $h \geq 0$. Set $h=12 s+l, 0 \leq l \leq 11$, and distinguish the following cases.

Case 1: $r=0,3$ and $l=1$ (odd $u$ ).

Write $\left\langle Z_{u} \cup X, D_{u}\right\rangle=\left\langle Z_{u} \cup\{\infty\},\{6 s+2\}\right\rangle \cup\left\langle Z_{u} \cup(X \backslash\{\infty\}), D^{\prime}\right\rangle \cup\left\langle Z_{u}, L\right\rangle$, where $D^{\prime}=[6 s+1,12 k+r+6 s+1] \backslash\{6 s+2\},\left|D^{\prime}\right|=12 k+r$, and $L=[1,6 s]$, and apply Lemmas 2.10, 2.14 and 2.15.

Case 2: $r=0,3,4,7$ and $l=9($ odd $u)$.

Write

$$
\begin{aligned}
&\left\langle Z_{u} \cup X, D_{u}\right\rangle=\left\langle Z_{u} \cup\left\{\infty_{1}, \infty_{2}, \infty_{3}\right\},\{1,6 s+3,6 s+4\}\right\rangle \cup \\
&\left\langle Z_{u} \cup\left\{\infty_{4}, \infty_{5}, \infty_{6}\right\},\{2,6 s+5,6 s+7\}\right\rangle \cup \\
&\left\langle Z_{u} \cup\left(X \backslash\left\{\infty_{1}, \infty_{2}, \ldots, \infty_{6}\right\}\right), D^{\prime}\right\rangle \cup\left\langle Z_{u}, L\right\rangle,
\end{aligned}
$$

where $D^{\prime}=[6 s+6,12 k+r+6 s+5] \backslash\{6 s+7\},\left|D^{\prime}\right|=12 k+r-1$, and $L=[3,6 s+2]$, and apply Lemmas 2.13, 2.14 and 2.15.

Case 3: $r=4^{\star}, 7$ and $l=5$ (odd $\left.u\right)$.

Write

$$
\begin{aligned}
& \left\langle Z_{u} \cup X, D_{u}\right\rangle=\left\langle Z_{u} \cup\left\{\infty_{1}, \infty_{2}, \infty_{3}, \infty_{4}\right\},\{2,4\}\right\rangle \cup\left\langle Z_{u} \cup\left\{\infty_{5}\right\},\{1\}\right\rangle \cup \\
& \left\langle Z_{u} \cup\left\{\infty_{6}\right\},\{6 s+8\}\right\rangle \cup\left\langle Z_{u} \cup\left(X \backslash\left\{\infty_{1}, \infty_{2}, \ldots, \infty_{6}\right\}\right), D^{\prime}\right\rangle \cup\left\langle Z_{u}, L\right\rangle,
\end{aligned}
$$

where $D^{\prime}=[6 s+3,12 k+r+6 s+3] \backslash\{6 s+4,6 s+8\},\left|D^{\prime}\right|=12 k+r-1$, and $L=[3,6 s+4] \backslash\{4,6 s+3\}$, and apply Lemmas 2.3, 2.10, 2.14 and 2.15.

Case 4: $r=0,4$ and $l=6$ (even $u$ ).

Write

$$
\begin{aligned}
&\left\langle Z_{u} \cup X, D_{u}\right\rangle=\left\langle Z_{u} \cup\left\{\infty_{1}, \infty_{2}, \infty_{3}\right\},\left\{1, \frac{u}{2}\right\}\right\rangle \cup \\
&\left\langle Z_{u} \cup\left\{\infty_{4}, \infty_{5}, \infty_{6}\right\},\{2,6 s+3,6 s+5\}\right\rangle \cup \\
&\left\langle Z_{u} \cup\left(X \backslash\left\{\infty_{1}, \infty_{2}, \ldots, \infty_{6}\right\}\right), D^{\prime}\right\rangle \cup\left\langle Z_{u}, L\right\rangle,
\end{aligned}
$$

where $D^{\prime}=[6 s+4,12 k+r+6 s+3] \backslash\{6 s+5\},\left|D^{\prime}\right|=12 k+r-1$, and $L=[3,6 s+2]$, and apply Lemmas 2.5, 2.13, 2.14 and 2.15.

Case 5: $r=0$ and $l=10$ (even $u$ ).

Write

$$
\begin{gathered}
\left\langle Z_{u} \cup X, D_{u}\right\rangle=\left\langle Z_{u} \cup\left\{\infty_{1}, \infty_{2}, \ldots, \infty_{6}\right\},\left\{1, \frac{u}{2}\right\}\right\rangle \cup \\
\left\langle Z_{u} \cup\left\{\infty_{7}, \infty_{8}, \infty_{9}, \infty_{10}\right\},\{2\}\right\rangle \cup \\
\left\langle Z_{u} \cup\left\{\infty_{11}\right\},\{4,6 s+3,6 s+5,6 s+6,6 s+7\}\right\rangle \cup \\
\left\langle Z_{u} \cup\left(X \backslash\left\{\infty_{1}, \infty_{2}, \ldots, \infty_{11}\right\}\right), D^{\prime}\right\rangle \cup\left\langle Z_{u}, L\right\rangle,
\end{gathered}
$$

where $D^{\prime}=[6 s+8,12 k+6 s+5],\left|D^{\prime}\right|=12 k-2$, and $L=[3,6 s+4] \backslash\{4,6 s+3\}$, and apply Lemmas 2.7, 2.2, 2.11, 2.14 and 2.15. 
Case 6: $r=3,7$ and $l=0$ (even $u)$.

Write

$$
\begin{aligned}
\left\langle Z_{u} \cup X, D_{u}\right\rangle=\left\langle Z_{u} \cup\left\{\infty_{1}, \infty_{2}, \ldots, \infty_{6}\right\},\left\{1, \frac{u}{2}\right\}\right\rangle \cup & \\
& \left\langle Z_{u} \cup\left(X \backslash\left\{\infty_{1}, \infty_{2}, \ldots, \infty_{6}\right\}\right), D^{\prime}\right\rangle \cup\left\langle Z_{u}, L\right\rangle,
\end{aligned}
$$

where $D^{\prime}=\{2\} \cup[6 s+3,12 k+r+6 s],\left|D^{\prime}\right|=12 k+r-1$, and $L=[3,6 s+2]$, and apply Lemmas 2.7, 2.14 and 2.15.

Case 7: $r=3$ and $l=4$ (even $u$ ).

Write

$$
\begin{aligned}
& \left\langle Z_{u} \cup X, D_{u}\right\rangle=\left\langle Z_{u} \cup\left\{\infty_{1}, \infty_{2}, \infty_{3}, \infty_{4}\right\},\left\{1, \frac{u}{2}\right\}\right\rangle \cup\left\langle Z_{u} \cup\left\{\infty_{5}\right\},\{2\}\right\rangle \cup \\
& \left\langle Z_{u} \cup\left\{\infty_{6}\right\},\{6 s+5\}\right\rangle \cup\left\langle Z_{u} \cup\left(X \backslash\left\{\infty_{1}, \infty_{2}, \ldots, \infty_{6}\right\}\right), D^{\prime}\right\rangle \cup\left\langle Z_{u}, L\right\rangle,
\end{aligned}
$$

where $D^{\prime}=[6 s+3,12 k+6 s+5] \backslash\{6 s+5\},\left|D^{\prime}\right|=12 k+2$, and $L=[3,6 s+2]$, and apply Lemmas 2.6, 2.10, 2.14 and 2.15.

Case 8: $r=4$ and $l=2$ (even $u$ ).

Write

$$
\begin{array}{r}
\left\langle Z_{u} \cup X, D_{u}\right\rangle=\left\langle Z_{u} \cup\left\{\infty_{1}, \infty_{2}, \infty_{3}, \infty_{4}\right\},\left\{1, \frac{u}{2}\right\}\right\rangle \cup\left\langle Z_{u} \cup\left\{\infty_{5}, \infty_{6}\right\},\{2\}\right\rangle \cup \\
\left\langle Z_{u} \cup\left(X \backslash\left\{\infty_{1}, \infty_{2}, \ldots, \infty_{6}\right\}\right), D^{\prime}\right\rangle \cup\left\langle Z_{u}, L\right\rangle,
\end{array}
$$

where $D^{\prime}=[6 s+3,12 k+6 s+5],\left|D^{\prime}\right|=12 k+3$, and $L=[3,6 s+2]$, and apply Lemmas 2.6, 2.1, 2.14 and 2.15.

Case 9: $r=7$ and $l=8$ (even $u$ ).

Write

$$
\begin{aligned}
&\left\langle Z_{u} \cup X, D_{u}\right\rangle=\left\langle Z_{u} \cup\left\{\infty_{1}, \infty_{2}, \infty_{3}\right\},\left\{1,2, \frac{u}{2}\right\}\right\rangle \cup \\
&\left\langle Z_{u} \cup\left\{\infty_{4}, \infty_{5}, \infty_{6}\right\},\{4,6 s+3,6 s+7\}\right\rangle \cup \\
&\left\langle Z_{u} \cup\left(X \backslash\left\{\infty_{1}, \infty_{2}, \ldots, \infty_{6}\right\}\right), D^{\prime}\right\rangle \cup\left\langle Z_{u}, L\right\rangle,
\end{aligned}
$$

where $D^{\prime}=[6 s+5,12 k+6 s+11] \backslash\{6 s+7\},\left|D^{\prime}\right|=12 k+6$, and $L=[3,6 s+4] \backslash$ $\{4,6 s+3\}$, and apply Lemmas 2.9, 2.13, 2.14 and 2.15.

Proposition 3.4. For any $n=60 k+5 r+2, r=2,7,10,11$, there exists a decomposition of $K_{n+u} \backslash K_{n}$ into 3 -suns for every admissible $u \geq 24 k+2 r+2$.

Proof. Let $X=\left\{\infty_{1}, \infty_{2}, \ldots, \infty_{60 k+5 r+2}\right\}, r=2,7,10,11$, and $u=24 k+2 r+2+h$, with $h \geq 0$. Set $h=12 s+l, 0 \leq l \leq 11$, and distinguish the following cases.

Case 1: $r=2,11$ and $l=3($ odd $u)$.

Write

$$
\begin{aligned}
&\left\langle Z_{u} \cup X, D_{u}\right\rangle=\left\langle Z_{u} \cup\left\{\infty_{1}\right\},\{6 s+2\}\right\rangle \cup\left\langle Z_{u} \cup\left\{\infty_{2}\right\},\{6 s+4\}\right\rangle \cup \\
&\left\langle Z_{u} \cup\left(X \backslash\left\{\infty_{1}, \infty_{2}\right\}\right), D^{\prime}\right\rangle \cup\left\langle Z_{u}, L\right\rangle,
\end{aligned}
$$


where $D^{\prime}=[6 s+1,12 k+r+6 s+2] \backslash\{6 s+2,6 s+4\},\left|D^{\prime}\right|=12 k+r$, and $L=[1,6 s]$, and apply Lemmas 2.10, 2.14 and 2.15.

Case 2: $r=2,7,10,11$ and $l=7($ odd $u)$.

Write

$$
\begin{gathered}
\left\langle Z_{u} \cup X, D_{u}\right\rangle=\left\langle Z_{u} \cup\left\{\infty_{1}, \infty_{2}\right\},\{1,2,6 s+3,6 s+4\}\right\rangle \cup \\
\left\langle Z_{u} \cup\left(X \backslash\left\{\infty_{1}, \infty_{2}\right\}\right), D^{\prime}\right\rangle \cup\left\langle Z_{u}, L\right\rangle,
\end{gathered}
$$

where $D^{\prime}=[6 s+5,12 k+r+6 s+4],\left|D^{\prime}\right|=12 k+r$, and $L=[3,6 s+2]$, and apply Lemmas 2.12, 2.14 and 2.15.

Case 3: $r=7,10$ and $l=11$ (odd $u$ ).

Write

$$
\begin{aligned}
& \left\langle Z_{u} \cup X, D_{u}\right\rangle=\left\langle Z_{u} \cup\left\{\infty_{1}, \infty_{2}, \infty_{3}\right\},\{1,6 s+3,6 s+4\}\right\rangle \cup \\
& \left\langle Z_{u} \cup\left\{\infty_{4}, \infty_{5}, \infty_{6}\right\},\{2,6 s+5,6 s+7\}\right\rangle \cup\left\langle Z_{u} \cup\left\{\infty_{7}\right\},\{6 s+8\}\right\rangle \cup \\
& \left\langle Z_{u} \cup\left(X \backslash\left\{\infty_{1}, \infty_{2}, \ldots, \infty_{7}\right\}\right), D^{\prime}\right\rangle \cup\left\langle Z_{u}, L\right\rangle,
\end{aligned}
$$

where $D^{\prime}=[6 s+6,12 k+r+6 s+6] \backslash\{6 s+7,6 s+8\},\left|D^{\prime}\right|=12 k+r-1$, and $L=[3,6 s+2]$, and apply Lemmas 2.13,2.10, 2.14 and 2.15.

Case 4: $r=2$ and $l=6$ (even $u$ ).

Write

$$
\begin{aligned}
&\left\langle Z_{u} \cup X, D_{u}\right\rangle=\left\langle Z_{u} \cup\left\{\infty_{1}, \infty_{2}, \infty_{3}, \infty_{4}\right\},\left\{1, \frac{u}{2}\right\}\right\rangle \cup \\
&\left\langle Z_{u} \cup\left\{\infty_{5}, \infty_{6}, \infty_{7}\right\},\{2,6 s+3,6 s+5\}\right\rangle \cup \\
&\left\langle Z_{u} \cup\left(X \backslash\left\{\infty_{1}, \infty_{2}, \ldots, \infty_{7}\right\}\right), D^{\prime}\right\rangle \cup\left\langle Z_{u}, L\right\rangle,
\end{aligned}
$$

where $D^{\prime}=[6 s+4,12 k+6 s+5] \backslash\{6 s+5\},\left|D^{\prime}\right|=12 k+1$, and $L=[3,6 s+2]$, and apply Lemmas 2.6, 2.13, 2.14 and 2.15.

Case 5: $r=2,10$ and $l=10$ (even $u)$.

Write

$$
\begin{aligned}
&\left\langle Z_{u} \cup X, D_{u}\right\rangle=\left\langle Z_{u} \cup\left\{\infty_{1}, \infty_{2}, \ldots, \infty_{6}\right\},\left\{1, \frac{u}{2}\right\}\right\rangle \cup \\
&\left\langle Z_{u} \cup\left\{\infty_{7}\right\},\{2,6 s+3,6 s+4,6 s+5,6 s+6\}\right\rangle \cup \\
&\left\langle Z_{u} \cup\left(X \backslash\left\{\infty_{1}, \infty_{2}, \ldots, \infty_{7}\right\}\right), D^{\prime}\right\rangle \cup\left\langle Z_{u}, L\right\rangle,
\end{aligned}
$$

where $D^{\prime}=[6 s+7,12 k+r+6 s+5],\left|D^{\prime}\right|=12 k+r-1$, and $L=[3,6 s+2]$, and apply Lemmas 2.7, 2.11, 2.14 and 2.15.

Case 6: $r=7,11$ and $l=4$ (even $u$ ).

Write

$$
\begin{aligned}
&\left\langle Z_{u} \cup X, D_{u}\right\rangle=\left\langle Z_{u} \cup\left\{\infty_{1}, \infty_{2}, \infty_{3}\right\},\left\{1, \frac{u}{2}\right\}\right\rangle \cup \\
&\left\langle Z_{u} \cup\left\{\infty_{4}, \infty_{5}, \infty_{6}, \infty_{7}\right\},\{2,4\}\right\rangle \cup \\
&\left.\quad Z_{u} \cup\left(X \backslash\left\{\infty_{1}, \infty_{2}, \ldots, \infty_{7}\right\}\right), D^{\prime}\right\rangle \cup\left\langle Z_{u}, L\right\rangle,
\end{aligned}
$$


where $D^{\prime}=[6 s+3,12 k+r+6 s+2] \backslash\{6 s+4\},\left|D^{\prime}\right|=12 k+r-1$, and $L=$ $[3,6 s+4] \backslash\{4,6 s+3\}$, and apply Lemmas 2.5, 2.3, 2.14 and 2.15.

Case 7: $r=7$ and $l=8$ (even $u$ ).

Write

$$
\begin{aligned}
& \left\langle Z_{u} \cup X, D_{u}\right\rangle=\left\langle Z_{u} \cup\left\{\infty_{1}, \infty_{2}, \infty_{3}\right\},\left\{1, \frac{u}{2}\right\}\right\rangle \cup \\
& \left\langle Z_{u} \cup\left\{\infty_{4}, \infty_{5}, \infty_{6}\right\},\{2,6 s+3,6 s+5\}\right\rangle \cup\left\langle Z_{u} \cup\left\{\infty_{7}\right\},\{6 s+7\}\right\rangle \cup \\
& \left\langle Z_{u} \cup\left(X \backslash\left\{\infty_{1}, \infty_{2}, \ldots, \infty_{7}\right\}\right), D^{\prime}\right\rangle \cup\left\langle Z_{u}, L\right\rangle,
\end{aligned}
$$

where $D^{\prime}=[6 s+4,12 k+6 s+11] \backslash\{6 s+5,6 s+7\},\left|D^{\prime}\right|=12 k+6$, and $L=[3,6 s+2]$, and apply Lemmas 2.5, 2.13, 2.10, 2.14 and 2.15.

Case 8: $r=10$ and $l=2($ even $u)$.

Write

$$
\begin{aligned}
\left\langle Z_{u} \cup X, D_{u}\right\rangle=\left\langle Z_{u} \cup\left\{\infty_{1}, \infty_{2}, \ldots, \infty_{6}\right\},\left\{1, \frac{u}{2}\right\}\right\rangle \cup\left\langle Z_{u} \cup\left\{\infty_{7}\right\},\{2\}\right\rangle \cup \\
\left\langle Z_{u} \cup\left(X \backslash\left\{\infty_{1}, \infty_{2}, \ldots, \infty_{7}\right\}\right), D^{\prime}\right\rangle \cup\left\langle Z_{u}, L\right\rangle,
\end{aligned}
$$

where $D^{\prime}=[6 s+3,12 k+6 s+11],\left|D^{\prime}\right|=12 k+9$, and $L=[3,6 s+2]$, and apply Lemmas 2.7, 2.10, 2.14 and 2.15.

Case 9: $r=11$ and $l=0($ even $u)$.

Write

$$
\begin{aligned}
\left\langle Z_{u} \cup X, D_{u}\right\rangle=\left\langle Z_{u} \cup\left\{\infty_{1}, \infty_{2}, \ldots, \infty_{7}\right\},\left\{1, \frac{u}{2}\right\}\right\rangle \cup & \\
& \left\langle Z_{u} \cup\left(X \backslash\left\{\infty_{1}, \infty_{2}, \ldots, \infty_{7}\right\}\right), D^{\prime}\right\rangle \cup\left\langle Z_{u}, L\right\rangle,
\end{aligned}
$$

where $D^{\prime}=\{2\} \cup[6 s+3,12 k+6 s+11],\left|D^{\prime}\right|=12 k+10$, and $L=[3,6 s+2]$, and apply Lemmas 2.8, 2.14 and 2.15.

Proposition 3.5. For any $n=60 k+5 r+3, r=2,5,6,9$, there exists a decomposition of $K_{n+u} \backslash K_{n}$ into 3 -suns for every admissible $u \geq 24 k+2 r+3$.

Proof. Let $X=\left\{\infty_{1}, \infty_{2}, \ldots, \infty_{60 k+5 r+3}\right\}, r=2,5,6,9$, and $u=24 k+2 r+3+h$, with $h \geq 0$. Set $h=12 s+l, 0 \leq l \leq 11$, and distinguish the following cases.

Case 1: $r=2,5,6,9$ and $l=4($ odd $u)$.

Write

$$
\begin{aligned}
\left\langle Z_{u} \cup X, D_{u}\right\rangle=\left\langle Z_{u} \cup\left\{\infty_{1}, \infty_{2}, \infty_{3}\right\}\right. & ,\{1,6 s+3,6 s+4\}\rangle \cup \\
& \left\langle Z_{u} \cup\left(X \backslash\left\{\infty_{1}, \infty_{2}, \infty_{3}\right\}\right), D^{\prime}\right\rangle \cup\left\langle Z_{u}, L\right\rangle,
\end{aligned}
$$

where $D^{\prime}=\{2\} \cup[6 s+5,12 k+r+6 s+3],\left|D^{\prime}\right|=12 k+r$, and $L=[3,6 s+2]$, and apply Lemmas 2.13, 2.14 and 2.15.

Case 2: $r=2,5$ and $l=8($ odd $u)$.

Write

$$
\begin{aligned}
\left\langle Z_{u} \cup X, D_{u}\right\rangle= & \left\langle Z_{u} \cup\left\{\infty_{1}, \infty_{2}\right\},\{1,6 s+3,6 s+4,6 s+5\}\right\rangle \cup \\
& \left\langle Z_{u} \cup\left\{\infty_{3}\right\},\{2\}\right\rangle \cup\left\langle Z_{u} \cup\left(X \backslash\left\{\infty_{1}, \infty_{2}, \infty_{3}\right\}\right), D^{\prime}\right\rangle \cup\left\langle Z_{u}, L\right\rangle,
\end{aligned}
$$


where $D^{\prime}=[6 s+6,12 k+r+6 s+5],\left|D^{\prime}\right|=12 k+r$, and $L=[3,6 s+2]$, and apply Lemmas 2.12, 2.10, 2.14 and 2.15.

Case 3: $r=6,9$ and $l=0($ odd $u)$.

If $s=0$, then write

$$
\begin{aligned}
\left\langle Z_{u} \cup X, D_{u}\right\rangle=\left\langle Z_{u} \cup\left\{\infty_{1}, \infty_{2}, \ldots, \infty_{8}\right\},\right. & \left.\left\{1, \frac{u}{3}\right\}\right\rangle \cup \\
& \left\langle Z_{u} \cup\left(X \backslash\left\{\infty_{1}, \infty_{2}, \ldots, \infty_{8}\right\}\right), D^{\prime}\right\rangle,
\end{aligned}
$$

where $D^{\prime}=[2,12 k+r+1] \backslash\left\{\frac{u}{3}\right\},\left|D^{\prime}\right|=12 k+r-1$, and apply Lemmas 2.4 and 2.14.

If $s>0$, then write

$$
\begin{aligned}
& \left\langle Z_{u} \cup X, D_{u}\right\rangle=\left\langle Z_{u} \cup\left\{\infty_{1}, \infty_{2}, \infty_{3}\right\},\{1,5 s, 5 s+1\}\right\rangle \cup \\
& \left\langle Z_{u} \cup\left\{\infty_{4}, \infty_{5}, \infty_{6}\right\},\{2,6 s+1,6 s+3\}\right\rangle \cup\left\langle Z_{u} \cup\left\{\infty_{7}\right\},\{6 s+2\}\right\rangle \cup \\
& \quad\left\langle Z_{u} \cup\left\{\infty_{8}\right\},\{6 s+4\}\right\rangle \cup\left\langle Z_{u} \cup\left(X \backslash\left\{\infty_{1}, \infty_{2}, \ldots, \infty_{8}\right\}\right), D^{\prime}\right\rangle \cup\left\langle Z_{u}, L\right\rangle,
\end{aligned}
$$

where $D^{\prime}=\{2 s+1,4 s\} \cup[6 s+5,12 k+r+6 s+1],\left|D^{\prime}\right|=12 k+r-1$, and $L=$ $[3,6 s] \backslash\{2 s+1,4 s, 5 s, 5 s+1\}$, and apply Lemmas 2.13, 2.10 and 2.14 to decompose the first five subgraphs, while to decompose the last one apply Lemma 2.15 i) and delete the orbit $\left(S_{0}\right)$.

Case 4: $r=2,6$ and $l=1$ (even $u$ ).

Write

$$
\begin{aligned}
\left\langle Z_{u} \cup X, D_{u}\right\rangle=\left\langle Z_{u} \cup\left\{\infty_{1}, \infty_{2}, \infty_{3}\right\}\right. & \left.,\left\{1, \frac{u}{2}\right\}\right\rangle \cup \\
& \left\langle Z_{u} \cup\left(X \backslash\left\{\infty_{1}, \infty_{2}, \infty_{3}\right\}\right), D^{\prime}\right\rangle \cup\left\langle Z_{u}, L\right\rangle,
\end{aligned}
$$

where $D^{\prime}=\{2\} \cup[6 s+3,12 k+r+6 s+1],\left|D^{\prime}\right|=12 k+r$, and $L=[3,6 s+2]$, and apply Lemmas 2.5, 2.14 and 2.15 .

Case 5: $r=2^{\star}$ and $l=5$ (even $u$ ).

Write

$$
\begin{aligned}
\left\langle Z_{u} \cup X, D_{u}\right\rangle=\left\langle Z_{u} \cup\left\{\infty_{1}, \infty_{2}, \ldots, \infty_{6}\right\},\left\{1, \frac{u}{2}\right\}\right\rangle \cup & \\
\left\langle Z_{u} \cup\left\{\infty_{7}, \infty_{8}, \infty_{9}, \infty_{10}\right\},\{2\}\right\rangle \cup & \\
\left\langle Z_{u} \cup\left\{\infty_{11}, \infty_{12}, \infty_{13}\right\},\{4,6 s+3,6 s+7\}\right\rangle \cup & \left\langle Z_{u} \cup\left(X \backslash\left\{\infty_{1}, \infty_{2}, \ldots, \infty_{13}\right\}\right), D^{\prime}\right\rangle \cup\left\langle Z_{u}, L\right\rangle,
\end{aligned}
$$

where $D^{\prime}=[6 s+5,12 k+6 s+5] \backslash\{6 s+7\},\left|D^{\prime}\right|=12 k$, and $L=[3,6 s+4] \backslash\{4,6 s+3\}$, and apply Lemmas 2.7, 2.2, 2.13, 2.14 and 2.15.

Case 6: $r=5,9$ and $l=7$ (even $u$ ).

Write

$$
\begin{aligned}
\left\langle Z_{u} \cup X, D_{u}\right\rangle= & \left\langle Z_{u} \cup\left\{\infty_{1}, \infty_{2}, \ldots, \infty_{6}\right\},\left\{1, \frac{u}{2}\right\}\right\rangle \cup \\
& \left\langle Z_{u} \cup\left\{\infty_{7}, \infty_{8}\right\},\{2,6 s+3,6 s+4,6 s+5\}\right\rangle \cup \\
& \left\langle Z_{u} \cup\left(X \backslash\left\{\infty_{1}, \infty_{2}, \ldots, \infty_{8}\right\}\right), D^{\prime}\right\rangle \cup\left\langle Z_{u}, L\right\rangle,
\end{aligned}
$$


where $D^{\prime}=[6 s+6,12 k+r+6 s+4],\left|D^{\prime}\right|=12 k+r-1$, and $L=[3,6 s+2]$, and apply Lemmas 2.7, 2.12, 2.14 and 2.15.

Case 7: $r=5$ and $l=11$ (even $u$ ).

Write

$$
\begin{aligned}
& \left\langle Z_{u} \cup X, D_{u}\right\rangle=\left\langle Z_{u} \cup\left\{\infty_{1}, \infty_{2}, \infty_{3}, \infty_{4}\right\},\left\{1, \frac{u}{2}\right\}\right\rangle \cup \\
& \left\langle Z_{u} \cup\left\{\infty_{5}, \infty_{6}\right\},\{2,6 s+3,6 s+5,6 s+6\}\right\rangle \cup\left\langle Z_{u} \cup\left\{\infty_{7}\right\},\{4\}\right\rangle \cup \\
& \quad\left\langle Z_{u} \cup\left\{\infty_{8}\right\},\{6 s+7\}\right\rangle \cup\left\langle Z_{u} \cup\left(X \backslash\left\{\infty_{1}, \infty_{2}, \ldots, \infty_{8}\right\}\right), D^{\prime}\right\rangle \cup\left\langle Z_{u}, L\right\rangle,
\end{aligned}
$$

where $D^{\prime}=[6 s+8,12 k+6 s+11],\left|D^{\prime}\right|=12 k+4$, and $L=[3,6 s+4] \backslash\{4,6 s+3\}$, and apply Lemmas 2.6, 2.12, 2.10, 2.14 and 2.15.

Case 8: $r=6$ and $l=9$ (even $u$ ).

Write

$$
\begin{aligned}
\left\langle Z_{u} \cup X, D_{u}\right\rangle & =\left\langle Z_{u} \cup\left\{\infty_{1}, \infty_{2}, \infty_{3}\right\},\left\{1, \frac{u}{2}\right\}\right\rangle \cup \\
& \left\langle Z_{u} \cup\left\{\infty_{4}, \infty_{5}, \infty_{6}\right\},\{2,6 s+3,6 s+5\}\right\rangle \cup\left\langle Z_{u} \cup\left\{\infty_{7}\right\},\{4\}\right\rangle \cup \\
& \left\langle Z_{u} \cup\left\{\infty_{8}\right\},\{6 s+7\}\right\rangle \cup\left\langle Z_{u} \cup\left(X \backslash\left\{\infty_{1}, \infty_{2}, \ldots, \infty_{8}\right\}\right), D^{\prime}\right\rangle \cup\left\langle Z_{u}, L\right\rangle,
\end{aligned}
$$

where $D^{\prime}=[6 s+6,12 k+6 s+11] \backslash\{6 s+7\},\left|D^{\prime}\right|=12 k+5$, and $L=[3,6 s+4] \backslash$ $\{4,6 s+3\}$, and apply Lemmas 2.5, 2.13, 2.10, 2.14 and 2.15.

Case 9: $r=9$ and $l=3$ (even $u$ ).

Write

$$
\begin{aligned}
\left\langle Z_{u} \cup X, D_{u}\right\rangle=\left\langle Z_{u} \cup\left\{\infty_{1}, \infty_{2}, \infty_{3}\right\}\right. & \left.,\left\{1,2, \frac{u}{2}\right\}\right\rangle \cup \\
& \left\langle Z_{u} \cup\left(X \backslash\left\{\infty_{1}, \infty_{2}, \infty_{3}\right\}\right), D^{\prime}\right\rangle \cup\left\langle Z_{u}, L\right\rangle,
\end{aligned}
$$

where $D^{\prime}=[6 s+3,12 k+6 s+11],\left|D^{\prime}\right|=12 k+9$, and $L=[3,6 s+2]$, and apply Lemmas 2.9, 2.14 and 2.15.

Proposition 3.6. For any $n=60 k+5 r+4, r=0,1,4,9$, there exists a decomposition of $K_{n+u} \backslash K_{n}$ into 3 -suns for every admissible $u \geq 24 k+2 r+3$.

Proof. Let $X=\left\{\infty_{1}, \infty_{2}, \ldots, \infty_{60 k+5 r+4}\right\}, r=0,1,4,9$, and $u=24 k+2 r+3+h$, with $h \geq 0$. Set $h=12 s+l, 0 \leq l \leq 11$, and distinguish the following cases.

Case 1: $r=0,1^{\star}, 4,9$ and $l=2($ odd $u)$.

Write

$$
\begin{aligned}
\left\langle Z_{u} \cup X, D_{u}\right\rangle=\left\langle Z_{u} \cup\left\{\infty_{1}, \infty_{2}, \infty_{3}, \infty_{4}\right\},\{2,4\}\right\rangle \cup & \\
& \left\langle Z_{u} \cup\left(X \backslash\left\{\infty_{1}, \infty_{2}, \infty_{3}, \infty_{4}\right\}\right), D^{\prime}\right\rangle \cup\left\langle Z_{u}, L\right\rangle,
\end{aligned}
$$

where $D^{\prime}=\{1,6 s+3\} \cup[6 s+5,12 k+r+6 s+2],\left|D^{\prime}\right|=12 k+r$, and $L=[3,6 s+$ $4] \backslash\{4,6 s+3\}$, and apply Lemmas 2.3, 2.14 and 2.15. 
Case 2: $r=0,9$ and $l=6($ odd $u)$.

Write

$$
\begin{aligned}
\left\langle Z_{u} \cup X, D_{u}\right\rangle & =\left\langle Z_{u} \cup\left\{\infty_{1}, \infty_{2}, \infty_{3}\right\},\{1,6 s+3,6 s+4\}\right\rangle \cup \\
& \left\langle Z_{u} \cup\left\{\infty_{4}\right\},\{2\}\right\rangle \cup\left\langle Z_{u} \cup\left(X \backslash\left\{\infty_{1}, \infty_{2}, \infty_{3}, \infty_{4}\right\}\right), D^{\prime}\right\rangle \cup\left\langle Z_{u}, L\right\rangle,
\end{aligned}
$$

where $D^{\prime}=[6 s+5,12 k+r+6 s+4],\left|D^{\prime}\right|=12 k+r$, and $L=[3,6 s+2]$, and apply Lemmas 2.13, 2.10, 2.14 and 2.15.

Case 3: $r=1,4$ and $l=10$ (odd $u)$.

Write

$$
\begin{aligned}
&\left\langle Z_{u} \cup X, D_{u}\right\rangle=\left\langle Z_{u} \cup\left\{\infty_{1}, \infty_{2}\right\},\{1,6 s+3,6 s+5,6 s+6\}\right\rangle \cup \\
&\left\langle Z_{u} \cup\left\{\infty_{3}\right\},\{2\}\right\rangle \cup\left\langle Z_{u} \cup\left\{\infty_{4}\right\},\{6 s+4\}\right\rangle \cup \\
&\left\langle Z_{u} \cup\left(X \backslash\left\{\infty_{1}, \infty_{2}, \infty_{3}, \infty_{4}\right\}\right), D^{\prime}\right\rangle \cup\left\langle Z_{u}, L\right\rangle,
\end{aligned}
$$

where $D^{\prime}=[6 s+7,12 k+r+6 s+6],\left|D^{\prime}\right|=12 k+r$, and $L=[3,6 s+2]$, and apply Lemmas 2.12, 2.10, 2.14 and 2.15.

Case 4: $r=0,4$ and $l=5$ (even $u$ ).

Write

$$
\begin{aligned}
&\left\langle Z_{u} \cup X, D_{u}\right\rangle=\left\langle Z_{u} \cup\left\{\infty_{1}, \infty_{2}, \ldots, \infty_{6}\right\},\left\{1, \frac{u}{2}\right\}\right\rangle \cup \\
&\left\langle Z_{u} \cup\left\{\infty_{7}, \infty_{8}, \infty_{9}\right\},\{2,6 s+3,6 s+5\}\right\rangle \cup \\
&\left\langle Z_{u} \cup\left(X \backslash\left\{\infty_{1}, \infty_{2}, \ldots, \infty_{9}\right\}\right), D^{\prime}\right\rangle \cup\left\langle Z_{u}, L\right\rangle,
\end{aligned}
$$

where $D^{\prime}=[6 s+4,12 k+r+6 s+3] \backslash\{6 s+5\},\left|D^{\prime}\right|=12 k+r-1$, and $L=[3,6 s+2]$, and apply Lemmas 2.7, 2.13, 2.14 and 2.15.

Case 5: $r=0$ and $l=9$ (even $u$ ).

Write

$$
\begin{aligned}
\left\langle Z_{u} \cup X, D_{u}\right\rangle & =\left\langle Z_{u} \cup\left\{\infty_{1}, \infty_{2}, \infty_{3}, \infty_{4}\right\},\left\{1, \frac{u}{2}\right\}\right\rangle \cup \\
& \left\langle Z_{u} \cup\left\{\infty_{5}, \infty_{6}, \infty_{7}\right\},\{2,6 s+3,6 s+5\}\right\rangle \cup\left\langle Z_{u} \cup\left\{\infty_{8}\right\},\{4\}\right\rangle \cup \\
& \left\langle Z_{u} \cup\left\{\infty_{9}\right\},\{6 s+7\}\right\rangle \cup\left\langle Z_{u} \cup\left(X \backslash\left\{\infty_{1}, \infty_{2}, \ldots, \infty_{9}\right\}\right), D^{\prime}\right\rangle \cup\left\langle Z_{u}, L\right\rangle,
\end{aligned}
$$

where $D^{\prime}=[6 s+6,12 k+6 s+5] \backslash\{6 s+7\},\left|D^{\prime}\right|=12 k-1$, and $L=[3,6 s+4] \backslash\{4,6 s+3\}$, and apply Lemmas $2.6,2.13,2.10,2.14$ and 2.15 .

Case 6: $r=1$ and $l=7$ (even $u$ ).

Write

$$
\begin{aligned}
&\left\langle Z_{u} \cup X, D_{u}\right\rangle=\left\langle Z_{u} \cup\left\{\infty_{1}, \infty_{2}, \ldots, \infty_{7}\right\},\left\{1, \frac{u}{2}\right\}\right\rangle \cup \\
&\left\langle Z_{u} \cup\left\{\infty_{8}, \infty_{9}\right\},\{2,4,6 s+3,6 s+5\}\right\rangle \cup \\
&\left\langle Z_{u} \cup\left(X \backslash\left\{\infty_{1}, \infty_{2}, \ldots, \infty_{9}\right\}\right), D^{\prime}\right\rangle \cup\left\langle Z_{u}, L\right\rangle,
\end{aligned}
$$

where $D^{\prime}=[6 s+6,12 k+6 s+5],\left|D^{\prime}\right|=12 k$, and $L=[3,6 s+4] \backslash\{4,6 s+3\}$, and apply Lemmas 2.8, 2.12, 2.14 and 2.15. 
Case 7: $r=1,9$ and $l=11($ even $u)$.

Write

$$
\begin{aligned}
\left\langle Z_{u} \cup X, D_{u}\right\rangle=\left\langle Z_{u} \cup\left\{\infty_{1}, \infty_{2}, \infty_{3}\right\},\left\{1, \frac{u}{2}\right\}\right\rangle \cup & \\
\left\langle Z_{u} \cup\left\{\infty_{4}\right\},\{2,4,6 s+3,6 s+5,6 s+6\}\right\rangle \cup & \left\langle Z_{u} \cup\left(X \backslash\left\{\infty_{1}, \infty_{2}, \infty_{3}, \infty_{4}\right\}\right), D^{\prime}\right\rangle \cup\left\langle Z_{u}, L\right\rangle,
\end{aligned}
$$

where $D^{\prime}=[6 s+7,12 k+r+6 s+6],\left|D^{\prime}\right|=12 k+r$, and $L=[3,6 s+4] \backslash\{4,6 s+3\}$, and apply Lemmas 2.5, 2.11, 2.14 and 2.15.

Case 8: $r=4$ and $l=1$ (even $u$ ).

Write

$$
\begin{aligned}
\left\langle Z_{u} \cup X, D_{u}\right\rangle=\left\langleZ _ { u } \cup \left\{\infty_{1}, \infty_{2},\right.\right. & \left.\left.\infty_{3}, \infty_{4}\right\},\left\{1, \frac{u}{2}\right\}\right\rangle \cup \\
& \left\langle Z_{u} \cup\left(X \backslash\left\{\infty_{1}, \infty_{2}, \infty_{3}, \infty_{4}\right\}\right), D^{\prime}\right\rangle \cup\left\langle Z_{u}, L\right\rangle,
\end{aligned}
$$

where $D^{\prime}=\{2\} \cup[6 s+3,12 k+6 s+5],\left|D^{\prime}\right|=12 k+4$, and $L=[3,6 s+2]$, and apply Lemmas 2.6, 2.14 and 2.15.

Case 9: $r=9$ and $l=3$ (even $u$ ).

Write

$$
\begin{aligned}
&\left\langle Z_{u} \cup X, D_{u}\right\rangle=\left\langle Z_{u} \cup\left\{\infty_{1}, \infty_{2}, \infty_{3}\right\},\left\{1, \frac{u}{2}\right\}\right\rangle \cup\left\langle Z_{u} \cup\left\{\infty_{4}\right\},\{2\}\right\rangle \cup \\
&\left\langle Z_{u} \cup\left(X \backslash\left\{\infty_{1}, \infty_{2}, \infty_{3}, \infty_{4}\right\}\right), D^{\prime}\right\rangle \cup\left\langle Z_{u}, L\right\rangle,
\end{aligned}
$$

where $D^{\prime}=[6 s+3,12 k+6 s+11],\left|D^{\prime}\right|=12 k+9$, and $L=[3,6 s+2]$, and apply Lemmas 2.5, 2.10, 2.14 and 2.15.

Combining Lemma 3.1 and Propositions 3.2-3.6 gives our main theorem.

Theorem 3.7. Any $3 \mathrm{SS}(n)$ can be embedded in a $3 \mathrm{SS}(m)$ if and only if

$$
m \geq \frac{7}{5} n+1 \quad \text { or } \quad m=n .
$$

\section{References}

[1] D. E. Bryant and C. A. Rodger, The Doyen-Wilson theorem extended to 5-cycles, J. Comb. Theory Ser. A 68 (1994), 218-225, doi:10.1016/0097-3165(94)90101-5.

[2] D. E. Bryant and C. A. Rodger, On the Doyen-Wilson theorem for $m$-cycle systems, J. Combin. Des. 2 (1994), 253-271, doi:10.1002/jcd.3180020405.

[3] J. Doyen and R. M. Wilson, Embeddings of Steiner triple systems, Discrete Math. 5 (1973), 229-239, doi:10.1016/0012-365x(73)90139-8.

[4] C.-M. Fu, Y.-L. Lin, S.-W. Lo, Y.-F. Hsu and W.-C. Huang, The Doyen-Wilson theorem for bull designs, Discrete Math. 313 (2013), 498-507, doi:10.1016/j.disc.2012.11.023.

[5] H.-L. Fu and C. C. Lindner, The Doyen-Wilson theorem for maximum packings of $K_{n}$ with 4-cycles, Discrete Math. 183 (1998), 103-117, doi:10.1016/s0012-365x(97)00080-0.

[6] H.-L. Fu, C. C. Lindner and C. A. Rodger, Two Doyen-Wilson theorems for maximum packings with triples, Discrete Math. 178 (1998), 63-71, doi:10.1016/s0012-365x(97)81817-1. 
[7] H. Gao and J. Wang, Doyen-Wilson theorem for perfect hexagon triple systems, Discrete Math. 311 (2011), 1006-1014, doi:10.1016/j.disc.2011.03.004.

[8] W.-C. Huang and W.-C. Yang, The Doyen-Wilson theorem for extended directed triple systems, Ars Combin. 84 (2007), 77-83.

[9] G. Lo Faro and A. Tripodi, The Doyen-Wilson theorem for kite systems, Discrete Math. 306 (2006), 2695-2701, doi:10.1016/j.disc.2006.03.074.

[10] G. Lo Faro and A. Tripodi, Embeddings of $\lambda$-fold kite systems, $\lambda \geq 2$, Australas. J. Combin. 36 (2006), 143-150, https://ajc.maths.uq.edu.au/pdf/36/ajc_v36_p143. pdf.

[11] R. Peltesohn, Eine Lösung der beiden Heffterschen Differenzenprobleme, Compositio Math. 6 (1939), 251-257, http://www.numdam.org/item?id=CM_1939_6_251_0.

[12] G. Stern and H. Lenz, Steiner triple systems with given subspaces; another proof of the DoyenWilson-theorem, Boll. Un. Mat. Ital. A Serie 517 (1980), 109-114.

[13] J. Wang, Perfect dexagon triple systems with given subsystems, Discrete Math. 309 (2009), 2930-2933, doi:10.1016/j.disc.2008.07.004.

[14] J.-X. Yin and B.-S. Gong, Existence of $G$-designs with $|V(G)|=6$, in: W. D. Wallis, H. Shen, W. Wei and L. Zhu (eds.), Combinatorial Designs and Applications, Marcel Dekker, New York, volume 126 of Lecture Notes in Pure and Applied Mathematics, 1990 pp. 201-218. 


\section{Appendix}

- $n=21, u=12 s+15$

Write

$$
\begin{gathered}
\left\langle Z_{u} \cup X, D_{u}\right\rangle=\left\langle Z_{u} \cup\left\{\infty_{1}, \infty_{2}, \infty_{3}, \infty_{4}\right\},\{2,4\}\right\rangle \cup \\
\left\langle Z_{u} \cup\left\{\infty_{5}\right\},\{1\}\right\rangle \cup\left\langle Z_{u} \cup\left\{\infty_{6}\right\},\{6 s+7\}\right\rangle \cup \\
\left\langle Z_{u} \cup\left(X \backslash\left\{\infty_{1}, \infty_{2}, \ldots, \infty_{6}\right\}\right),\{6 s+3,6 s+5,6 s+6\}\right\rangle \cup\left\langle Z_{u}, L\right\rangle,
\end{gathered}
$$

where $L=[3,6 s+4] \backslash\{4,6 s+3\}$, and apply Lemmas 2.3, 2.10, 2.14 and 2.15.

- $n=13, u=12 s+12$

Write

$$
\begin{aligned}
\left\langle Z_{u} \cup X, D_{u}\right\rangle=\left\langle Z_{u} \cup\left\{\infty_{1}, \infty_{2}, \ldots, \infty_{6}\right\},\{1,6 s+6\}\right\rangle \cup & \\
& \left\langle Z_{u} \cup\left\{\infty_{7}, \infty_{8}, \infty_{9}, \infty_{10}\right\},\{2\}\right\rangle \cup \\
& \left\langle Z_{u} \cup\left\{\infty_{11}, \infty_{12}, \infty_{13}\right\},\{4,6 s+3,6 s+5\}\right\rangle \cup\left\langle Z_{u}, L\right\rangle,
\end{aligned}
$$

where $L=[3,6 s+4] \backslash\{4,6 s+3\}$, and apply Lemmas 2.7, 2.2, 2.13 and 2.15.

- $n=9, u=12 s+7$

Write

$$
\begin{aligned}
\left\langle Z_{u} \cup X, D_{u}\right\rangle=\left\langle Z_{u} \cup\left\{\infty_{1}, \infty_{2}, \infty_{3}, \infty_{4}\right\},\{2,4\}\right\rangle \cup & \\
& \left\langle Z_{u} \cup\left\{\infty_{5}, \infty_{6}, \infty_{7}, \infty_{8}, \infty_{9}\right\},\{1\}\right\rangle \cup\left\langle Z_{u}, L\right\rangle,
\end{aligned}
$$

where $L=[3,6 s+3] \backslash\{4\}$, and apply Lemmas 2.3, 2.14 and decompose $\left\langle Z_{u}, L\right\rangle$ as in Lemma 2.15 iii), taking in account that $|6 s+4|_{12 s+7}=6 s+3$. 